\title{
Continua of states in boundary-layer flows
}

Hewitt, R.E. and Duck, P.W and Stow, S.R 2002

Manchester Institute for Mathematical Sciences

School of Mathematics

The University of Manchester

\footnotetext{
Reports available from: http://eprints.maths.manchester.ac.uk/

And by contacting: The MIMS Secretary

School of Mathematics

The University of Manchester

Manchester, M13 9PL, UK
} 


\title{
Continua of states in boundary-layer flows
}

\author{
By R. E. HEWITT $\dagger$, P. W. DUCK AND S. R. STOW \\ Department of Mathematics, University of Manchester, Oxford Road, Manchester, M13 9PL, UK
}

(Received 5 July 2001 and in revised form 11 March 2002)

We consider a class of three-dimensional boundary-layer flows, which may be viewed as an extension of the Falkner-Skan similarity form, to include a cross-flow velocity component, about a plane of symmetry. In general, this provides a range of threedimensional boundary-layer solutions, parameterized by a Falkner-Skan similarity parameter, $n$, together with a further parameter, $\Psi_{\infty}$, which is associated with a cross-flow velocity component in the external flow. In this work two particular cases are of special interest: for $n=0$ the similarity equations possess a family of solutions related to the Blasius boundary layer; for $n=1$ the similarity solution provides an exact reduction of the Navier-Stokes equations corresponding to the flow near a saddle point of attachment. It is known from the work of Davey (1961) that in this latter class of flow, a continuum of solutions can be found. The continuum arises (in general) because it is possible to find states with an algebraic, rather than exponential, behaviour in the far field. In this work we provide a detailed overview of the continuum states, and show that a discrete infinity of 'exponential modes' are smoothly embedded within the 'algebraic modes' of the continuum. At a critical value of the cross-flow, these exponential modes appear as a cascade of eigensolutions to the far-field equations, which arise in a manner analogous to the energy eigenstates found in quantum mechanical problems described by the Schrödinger equation.

The presence of a discrete infinity of exponential modes is shown to be a generic property of the similarity equations derived for a general $n$. Furthermore, we show that there may also exist non-uniqueness of the continuum; that is, more than one continuum of states can exist, that are isolated for fixed $n$ and $\Psi_{\infty}$, but which are connected through an unfolded transcritical bifurcation at a critical value of the cross-flow parameter, $\Psi_{\infty}$.

The multiplicity of states raises the question of solution selection, which is addressed using two stability analyses that assume the same basic symmetry properties as the base flow. In one case we consider a steady, algebraic form in the 'streamwise' direction, whilst in the other a temporal form is assumed. In both cases it is possible to extend the analysis to consider a continuous spectrum of disturbances that decay algebraically in the wall-normal direction. We note some obvious parallels that exist between such stability analyses and the approach to the continua of states described earlier in the paper.

We also discuss the appearance of analogous non-unique states to the Falkner-Skan equation in the presence of an adverse pressure gradient (i.e. $n<0)$ in an appendix.

\footnotetext{
$\dagger$ Manchester Centre for Nonlinear Dynamics.

† Current address: Department of Engineering, University of Cambridge, Trumpington Street, Cambridge CB2 1PZ, UK.
} 


\section{Introduction}

The flow of a viscous incompressible fluid near a stagnation point of attachment is a fundamental problem in fluid mechanics. Not only is the flow near such points of significant practical importance, it is also one of the rare instances in which a similarity form may be constructed that allows an exact reduction of the NavierStokes equations to an ordinary-differential system.

Of particular relevance to this current discussion is the previous work of Howarth (1951), Davey (1961), Davey \& Schofield (1967) and Schofield \& Davey (1967). In all these works, a class of solution is sought, in a Cartesian coordinate system $(x, y, z)$, of the form

$$
u^{*}=a x f^{\prime}(\eta), \quad v^{*}=-v^{1 / 2}[a f(\eta)+b g(\eta)] / a^{1 / 2}, \quad w^{*}=b z g^{\prime}(\eta),
$$

where $\eta=a^{1 / 2} y / v^{1 / 2}$ and $v$ is the kinematic viscosity of the fluid. Here $x$ and $z$ are coordinates measured parallel to the planar boundary and $y$ is measured perpendicular to the boundary. The velocity profiles $f$ and $g$ satisfy a sixth-order boundary-value problem parameterized by $c=b / a$, with four boundary conditions at the plane $y=0$ corresponding to no-slip and impermeability. The external flow field is of the form $\{a x,-(a+b)(y-\delta), b z\}$ where $a(>0)$ and $b$ are constants and $\delta$ is a displacement thickness; thus there are two additional conditions, namely that $f^{\prime} \rightarrow 1$ and $g^{\prime} \rightarrow 1$ as $\eta \rightarrow \infty$.

This form of solution is of obvious practical relevance for a flow over a smooth body, describing as it does the flow in a local tangent plane near a stagnation streamline. The point of significant mathematical and physical interest is that a continuum of solutions to the sixth-order boundary-value problem for $f$ and $g$ can be constructed for a range of the parameter $c$, as discovered by the analysis of Davey (1961), which extended the previous results of Howarth (1951). Davey noted that the existence of a continuum of states may be associated with modes that approach the far-field behaviour algebraically fast, rather than exponentially. We note that a less careful analysis than that provided by Davey could easily miss the presence of the continuum, since iterative numerical approaches that apply point conditions at some outer edge, $\eta=\eta_{\infty}$, will artificially select only those modes that decay exponentially if the algebraic component of decay is relatively slow. Nevertheless, Davey (1961), followed later by Davey \& Schofield (1967) and Schofield \& Davey (1967) made, in the current authors' opinion, a somewhat arbitrary decision in selecting only those modes that have some degree of exponential approach to the correct edge conditions, thereby removing the continuum (indeed, this provides the motivation for the work of $\S 6$, in which stability issues are addressed). Furthermore, in the case $c=-1$ (or $\Psi_{\infty}=2$, in the notation of the work we shall present here) the otherwise algebraic modes of the continuum become exponential (We classify any solution that has a far-field decay in the form $F(\eta) \mathrm{e}^{H(\eta)}$ (for algebraic functions $F(\eta)$ and $H(\eta)$ ), as an exponential mode.) In the work of Davey, and the subsequent analyses co-authored by Davey and Schofield, an extra condition is applied, namely that the 'appropriate' solution within the continuum is the state that approaches the far-field conditions most rapidly, i.e. the fastest spatially decaying exponential mode. There is clearly no $a$ priori reason for making this selection, as has been noted in a more recent discussion of a related problem given by Hall, Balakumar \& Papageorgiou (1992). One of the aims of this paper is to shed some light on this choice; this aspect motivates our discussion of stability issues as given in $\S 6$.

Having decided to discard those states that decay to the appropriate far-field 
behaviour algebraically, Davey \& Schofield (1967) and Schofield \& Davey (1967) then find two branches of exponential modes. This second branch of solutions leads to the interesting result that a fully three-dimensional alternative state exists even for the axisymmetric external flow field of $c=1\left(\Psi_{\infty}=-2\right.$ in the notation of this paper). The existence of alternative solutions of this type was later extended to provide (exact) non-axisymmetric flows of von Kármán type by Hewitt, Duck \& Foster (1999) and Hewitt \& Duck (2000). As we shall show, the two solution branches described by the work of Davey and Schofield are in fact just the first two of a countably infinite sequence of states that have an exponential decay to the free-stream conditions.

The presence of a continuum of algebraically decaying states is clearly worth further comment. In this regard we note that there is an argument for discarding such solutions in a boundary-layer model. Goldstein (1965) argues that one must allow only for an exponential approach to the outer-flow conditions. This argument is applicable only when the flow in question is free of vorticity in the free stream, and allows one to rule out algebraic decay within the boundary layer, since this would otherwise require solutions of the inviscid flow to become singular (behaving as $y^{-\alpha}$, for some $\alpha>0$ ) on approaching the boundary (as $y \rightarrow 0$ ). Such a singular behaviour is contrary to the known properties of harmonic functions. The saddle point of attachment is, however, an exact reduction of the Navier-Stokes equations, which although of boundary-layer type, requires no formal approximation. In this regard, we may point out that the work of Davey and Schofield is formally correct in their concentration on the 'exponential' modes, since an a priori decision is made to consider only irrotational 'external' flow. Nevertheless, the continuum of algebraic modes correspond to an exact solution with an algebraically decaying component of vorticity. Therefore algebraic boundary-layer flows are clearly acceptable solutions if one is prepared to relax the condition of a potential external flow and permit, instead, the presence of free-stream vorticity. We also note that a closely related analysis of the role of free-stream vorticity has been performed by Dhanak \& Stuart (1995). However, their analysis corresponds to a discussion of eigensolutions of the disturbance vorticity equations that grow algebraically away from the wall and match to an external flow that has weak cross-stream vorticity; we note also that their free-stream vortical contribution was everywhere of small amplitude. The physical motivation of their study is that free-stream vorticity may be sufficiently amplified in the stagnation-point flow to significantly alter the transition process.

The situation for boundary layers is somewhat more subtle than suggested by Goldstein (1965), as has been discussed in the work of Brown \& Stewartson (1965), which describes how similarity solutions, in which there is an algebraic decay of vorticity, can be realized as limit solutions of the full (two-dimensional) boundarylayer equations with exponential decay. Several classes of two-dimensional boundarylayer problems were studied by these latter authors. In the case of Falkner-Skan-type problems, algebraically decaying solutions were shown to occur only for negative values of the Hartree parameter $\beta$ (denoted as $\lambda$ by some authors or $-1<n<0$ in the notation of the present paper, since $\beta=2 n /(1+n))$, corresponding to what may be regarded as adverse-pressure-gradient boundary layers. Algebraically decaying solutions were also found to occur in two other (non-similar) forms. Further comment regarding the Falkner-Skan equation, and its connection with the flows described herein is given in the Appendix. We note also the work of Ackerberg (1965) on the draining flow from the apex of an inverted cone. In the high-Reynolds-number limit of this problem, it was found that algebraically decaying components of the 
boundary-layer solution (on the cone walls) were essential for the proper matching with the core of the flow.

More recently, some other three-dimensional (exponentially decaying), similaritytype boundary-layer solutions have been described. In seeking solutions to corner boundary-layer problems, Ridha (1992) and Dhanak \& Duck (1997) found that if a cross-flow component of flow is permitted, which grows linearly in the cross-flow direction (the other two velocity components are independent of this distance), then two distinct solution branches are obtained. A corollary which arises from this work is that the (two-dimensional) Blasius solution has a three-dimensional alternative, which possesses a jet-like cross-flow velocity component. This aspect was discussed by Duck, Stow \& Dhanak (2000), who were able to show that in the context of flat-plate (similarity-type) boundary layers, in addition to the classical Falkner-Skan solution branch, a further three-dimensional branch also exists. In fact for adverse-pressuregradient boundary layers (i.e. $n<0$ in our notation), there is a narrow range of $n$ for which six solutions are possible, all exponentially decaying in the far field. An important point to note here is that even though no cross-flow velocity component is forced through external/boundary conditions, nonetheless such a velocity component can occur (possessing a jet-like profile). Some added importance was given to these additional three-dimensional states by Duck, Stow \& Dhanak (1999, 2000), who showed that in some $(n)$ parameter regimes (including the $n=0$, Blasius state), the classical states may be regarded as being unstable, insofar as they are susceptible to eigenstates that grow algebraically in the streamwise direction; this aspect has been addressed in more detail by Duck \& Dry (2001), and an analysis of this type will be applied to the current solutions in $\S 6$.

In this paper we seek similarity solutions to the three-dimensional boundary-layer equations of the general form considered by Duck et al. (1999, 2000), but also allow for the existence of a free-stream cross-flow; as such our class of flows also incorporates that considered by Davey (1961), and if one were to include a rotational component could also capture the equations considered by Hewitt et al. (1999) and Hewitt \& Duck (2000). This leads to a nonlinear boundary-value problem, parameterized by the pressure gradient parameter $(n)$ and the measure of relative cross-flow in the external flow, denoted by $\Psi_{\infty}$. Furthermore, on setting $\Psi_{\infty}=1-n$, the solutions of the Falkner-Skan equations form a (zero cross-flow) subset of solutions to the current system. The analysis herein concentrates primarily on $n \geqslant 0$, although in the Appendix we discuss the influence of an adverse pressure gradient $(n<0)$ and make some connections between the current analysis and two-dimensional solutions of the Falkner-Skan equation. As we shall show, the apparently straightforward system of ordinary-differential equations that results from this class of similarity solution possesses some rather complicated and unsuspected features. Most significantly, we shall see that even restricting attention to those solutions that have exponential decay in the far field (as suggested by Davey and others) nevertheless yields a discrete set of infinitely many states (rather than just the two states discussed by Davey \& Schofield (1967)). This countably infinite set can be viewed as being smoothly embedded within the continuum of states noted by Davey (1961), and is a generic property of the similarity solutions for general values of $n$.

Furthermore, we shall see (with quantitative details provided in the particular case of $n=0$ ) that for a fixed cross-flow parameter in the external flow (i.e. for fixed values of $\Psi_{\infty}$ ) the continuum of solutions can itself be non-unique, in the sense that two continua exist that are isolated for fixed $\Psi_{\infty}$. On variation of $\Psi_{\infty}$ the continua will be shown to reconnect through the mechanism of an unfolded transcritical bifurcation. 
The format of this paper is as follows. In $\S 2$ we formulate a class of boundary-layer solutions that can be viewed as a three-dimensional extension of the Falkner-Skan family of states, valid near a plane of cross-flow symmetry. In $\S 3$ we give an overview of the numerical results, including details of those states with an exponential decay and those with an algebraic decay in the free stream. This section highlights a number of interesting solution features and motivates a detailed asymptotic investigation of the cascade of exponential states given in $\S 4$, as well as the additional asymptotic limits presented in $\S 5$. In $\S 6$, a stability analysis (linked to the general approach adopted throughout this paper) is presented and provides some preliminary results regarding which of the various solutions are likely to be realizable. Finally, in $\S 7$ we present some conclusions regarding the implications of this work.

\section{Formulation}

We seek a self-similar boundary-layer solution to the non-dimensionalized NavierStokes equations (in a Cartesian coordinate system $\{x, y, z\}$ ) of the form

$$
\begin{gathered}
u^{*}=x^{n} U(\eta)+\cdots, \quad v^{*}=\frac{x^{(n-1) / 2}}{\sqrt{2 R e}}[(1-n) \eta U(\eta)-\Phi(\eta)]+\cdots, \\
w^{*}=\frac{z x^{n-1}}{2}[(1-n) U(\eta)-\Psi(\eta)]+\cdots, \\
p^{*}=-\frac{x^{2 n} P_{1}}{2}-\frac{z^{2} x^{2 n-2} P_{2}}{2}+x^{n-1} \frac{Q(\eta, z)}{R e}+\cdots,
\end{gathered}
$$

where

$$
\eta=x^{(n-1) / 2} \sqrt{\frac{R e}{2}} y .
$$

Here, $\eta$ spans the boundary layer, and it is generally assumed that $|z| \ll 1$ (although in the case of $n=1$ this restriction proves not to be necessary). This solution may be regarded as relevant to flows in the neighbourhood of a plane of symmetry $(z=0)$. Here, $P_{1}, P_{2}$ are pressure constants and $R e$ is a Reynolds number based on the external dimensional 'streamwise' flow speed $U_{\infty}$.

It is clear that in the case $n=1$, there is a simple correlation between the $\{U, \Phi, \Psi\}$ above and $\left\{f^{\prime}, a f+b g, g^{\prime}\right\}$ in the formulation of equations (1.1) for the saddle point of attachment. Furthermore, in the case $n=1$, although the solution is of boundarylayer type, no corresponding approximation is required, and the solution is an exact reduction of the Navier-Stokes equations.

The Navier-Stokes system, after substitution of (2.1) and elimination of $Q(\eta, z)$ by cross-differentiation, is thus reduced, under a boundary-layer approximation, to

$$
\begin{gathered}
2 U=\Phi^{\prime}+\Psi, \\
U^{\prime \prime}+\Phi U^{\prime}-2 n U^{2}=-2 n, \\
\Psi^{\prime \prime}+\Phi \Psi^{\prime}+\Psi^{2}-\left(1-n^{2}\right) U^{2}=\Psi_{\infty}^{2}-\left(1-n^{2}\right) .
\end{gathered}
$$

The boundary conditions are simply no-slip and impermeability at the boundary

$$
U=\Phi=\Psi=0 \quad \text { on } \quad \eta=0,
$$

together with an approach to the external flow conditions

$$
U \rightarrow 1, \quad \Psi \rightarrow \Psi_{\infty} \quad \text { as } \quad \eta \rightarrow \infty .
$$


The system has two dimensionless parameters, the Falkner-Skan/pressure-gradient parameter $n$ and the measure of far-field cross-flow, as denoted by $\Psi_{\infty}$.

Clearly, solutions for which $\Psi(\eta) \equiv(1-n) U(\eta)$ reduce $(2.3 b)$ and $(2.3 c)$ to the same form and correspond to two-dimensional states with $w^{*} \equiv 0$ in $(2.1)$; this is the Falkner-Skan family of solutions. Similarly, the component of cross flow in the free stream is zero for $\Psi_{\infty}=1-n$, although, as shown by Duck et al. (2000), a free-stream cross-flow velocity component is not necessary to provoke a cross-flow velocity component within the boundary layer.

It is worth noting at this stage that although the problem (2.3), together with (2.4), is formally a boundary-value problem, it can be posed as a Cauchy problem. In particular, we note that there exists a simple scaling symmetry (when $n=0$ ) of the form

$$
U \rightarrow \frac{U}{\hat{c}}, \quad \Psi \rightarrow \frac{\Psi}{\hat{c}}, \quad \Phi \rightarrow \frac{\Phi}{\sqrt{\hat{c}}}, \quad \frac{\mathrm{d}}{\mathrm{d} \eta} \rightarrow \frac{1}{\sqrt{\hat{c}}} \frac{\mathrm{d}}{\mathrm{d} \eta}, \quad K \rightarrow \frac{K}{\hat{c}^{2}},
$$

where $K \equiv \Psi_{\infty}^{2}-1$, and $\hat{c}$ is a constant. Thus, we can pose the same system (2.3) with the initial conditions (by choosing $\hat{c}=\left[U^{\prime}(0)\right]^{2 / 3}$ )

$$
U(0)=\Phi(0)=\Psi(0)=0, \quad U^{\prime}(0)=1, \quad \Psi^{\prime}(0)=\Lambda,
$$

to provide a solution structure spanned by the two independent parameters $\Lambda$ and $K$.

For most of the subsequent analysis we shall prefer to describe the solution structure in terms of the original boundary-value problem; however there are some cases that can be most easily analysed in terms of this initial-value formulation.

\section{The large- $\eta$ behaviour}

The approach to the external flow conditions can be obtained by seeking a large- $\eta$ solution such that

$$
U=1+u(\eta), \quad \Phi=\left(2-\Psi_{\infty}\right) \eta+\delta^{*}+\phi(\eta), \quad \Psi=\Psi_{\infty}+\psi(\eta),
$$

where $\delta^{*}$ is the displacement thickness and

$$
\begin{aligned}
u^{\prime \prime}+\left[\left(2-\Psi_{\infty}\right) \eta+\delta^{*}\right] u^{\prime}-4 n u & =0, \\
\psi^{\prime \prime}+\left[\left(2-\Psi_{\infty}\right) \eta+\delta^{*}\right] \psi^{\prime}+2 \Psi_{\infty} \psi & =0 .
\end{aligned}
$$

Here we have implicitly assumed that $|u| \ll|w|$, which can be verified a posteriori to be consistent with the subsequent analysis. Solutions to $(2.8 a)$ can be given simply in terms of hypergeometric functions following the substitution

$$
\xi=\frac{\left(2-\Psi_{\infty}\right) \eta+\delta^{*}}{\sqrt{2-\Psi_{\infty}}},
$$

the standard leading-order asymptotic forms of which yield

$$
\begin{aligned}
& u=A_{1} \xi^{4 n /\left(2-\Psi_{\infty}\right)}+A_{2} \xi^{-1-4 n /\left(2-\Psi_{\infty}\right)} \mathrm{e}^{-\xi^{2} / 2}, \\
& \psi=A_{3} \xi^{-2 \Psi_{\infty} /\left(2-\Psi_{\infty}\right)}+A_{4} \xi^{-1+2 \Psi_{\infty} /\left(2-\Psi_{\infty}\right)} \mathrm{e}^{-\xi^{2} / 2},
\end{aligned}
$$

provided that $2-\Psi_{\infty}=O(1)$, where $A_{1,2,3,4}$ are constants.

The non-existence of solutions for $\Psi_{\infty}>2$ has been shown by Davey (1961), and therefore we restrict attention to $\Psi_{\infty} \leqslant 2$. Clearly $A_{1}=0$ to ensure decay in $u$ (for 
$n>0$, see also the Appendix), which (for $\Psi_{\infty} \geqslant 0$ ) leaves the four constants $A_{2}, A_{3}$, $A_{4}$ and $\delta^{*}$ that are subject to just three boundary conditions $(U=\Phi=\Psi=0$ at $\eta=0$ ). It is this freedom, arising from the possibility of an algebraic approach to the external flow (i.e. a non-zero $A_{3}$ ), that leads to continua of solutions. Alternatively, for $\Psi_{\infty}<0$ we must also require that $A_{3}=0$ thereby leading to just states with exponential decay and hence no continua.

We may also note in passing that continua states are also possible in the case $n<0, \Psi_{\infty}<0$, for which it is possible to find solutions with $A_{1} \neq 0$ and $A_{3}=0$. Clearly, there are also cases in which a two-dimensional continuum of states can (in general) be achieved with both $A_{1}$ and $A_{3}$ non-zero if $n<0$ and $\Psi_{\infty}>0$. Although some discussion of the two-dimensional (Falkner-Skan) states with $n<0$ (and $\Psi_{\infty}=1-n$ ) is given in the Appendix, we shall not consider the additional complication of both an adverse pressure gradient and three-dimensionality, concentrating instead on $n \geqslant 0$ (with particular emphasis on the important cases $n=0$ and $n=1)$.

\section{An overview of the solutions}

We begin our discussion regarding the solutions to (2.3) by presenting an overview of the numerical results. Computations were performed using two entirely independent methods: (i) a method analogous to that of Davey (1961) was applied, utilizing a fourth-order Runge-Kutta algorithm based upon the initial-value formulation arising from (2.5) and (2.6) (see Stow 1999); (ii) the boundary-value problem (2.3) was approached using the arclength continuation method implemented by AUTO. Clearly, some further explanation is required regarding exactly how the boundary conditions are applied in the boundary-value formulation. If one imposes the formal conditions (2.4), together with truncation of the domain at $\eta=\eta_{\infty}$, then the numerically realized solution is, in general, that for which $A_{3}=0$ in the far-field behaviour (2.10). This in fact provides a useful method of enabling a numerical continuation of the 'exponential modes' to be performed. An alternative approach is required to capture the algebraic decay in the far-field cross-flow velocity component. Given the extra degree of freedom provided by a non-zero $A_{3}$ in (2.10), one may additionally specify $U^{\prime}(0)$ or $\Psi^{\prime}(0)$. This allows computations of cross-sections of the continua of states, for which we can determine the functional dependence $A_{3}\left(\Psi_{\infty}\right)$. Thus the exponential modes may (also) be obtained by seeking the locus in parameter space of the roots $A_{3}\left(\Psi_{\infty}\right)=0$.

\subsection{The (exact) solution for $n=1$ : exponential modes}

Utilizing the procedures described above, it is possible to construct a view of the solutions in parameter space. For fixed values of $n$, one may consider the projections in the planes spanned by $\left\{U^{\prime}(0), \Psi_{\infty}\right\},\left\{\Psi^{\prime}(0), \Psi_{\infty}\right\}$ and $\left\{\delta^{*}, \Psi_{\infty}\right\}$. Clearly, for fixed $n$ a solution to (2.3) is uniquely defined at any point in a three-dimensional parameter space spanned by $U^{\prime}(0), \Psi^{\prime}(0)$ and $\Psi_{\infty}$. Nevertheless, $\delta^{*}$ plays a significant role in the asymptotic descriptions to be presented later, and is hence included here.

In figure 1, we show the branches of exponential modes to (2.3) over a range of $\Psi_{\infty} \in[-2,2]$. At the two extremes of this range, the external flow configurations are an axisymmetric nodal flow for $\Psi_{\infty}=-2$ and a saddle-point flow for $\Psi_{\infty}=2$. As noted earlier, Davey (1961) showed that solutions cannot be found for $\Psi_{\infty}>2$. Solutions can be found for $\Psi_{\infty}<-2$, but can be simply transformed into the range $\Psi_{\infty}>-2$ as noted by Davey \& Schofield (1967). The two main solutions (branches 


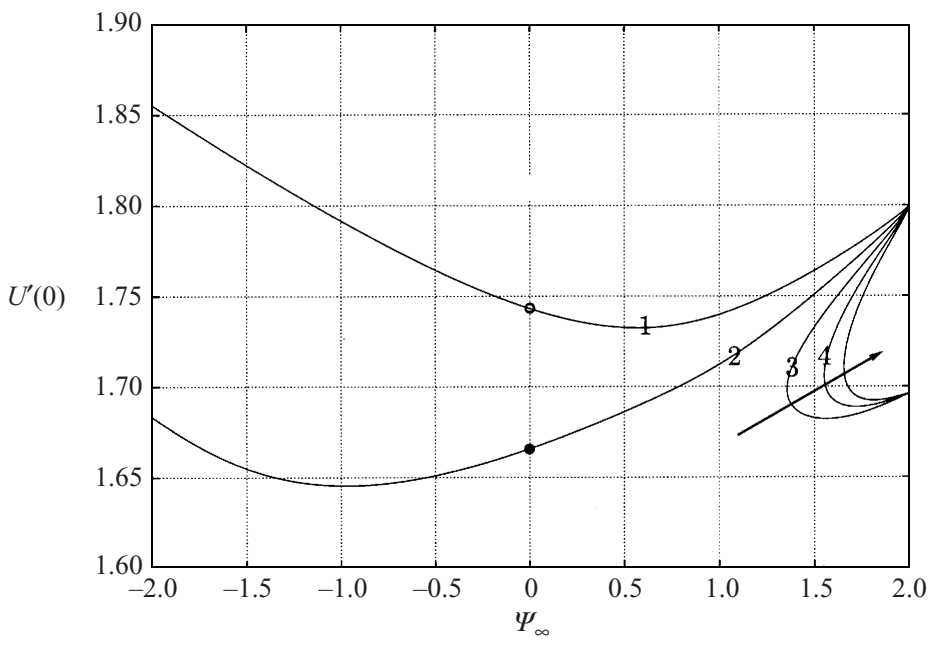

FiguRE 1. Properties of the 'exponential modes' for varying external cross-flow, $\Psi_{\infty}$ and $n=1$. The open circle denotes the two-dimensional Hiemenz solution with $\Psi(\eta) \equiv 0$, and the filled circle denotes the three-dimensional alternative solution in the second branch of states described by Davey \& Schofield (1967). The arrow indicates that there are further solution branches beyond those shown (as $\Psi_{\infty} \rightarrow 2$ ).

1 and 2) that cover the entire range of $\Psi_{\infty}$ are the dual solutions described in the two papers of Davey and Schofield. However, it is readily observed that additional states can be obtained in the limit of $\Psi_{\infty} \rightarrow 2$. The arrow in figures 1 and 3 indicates the presence of a cascade of such states, the first few of which are presented in those data.

We note that this non-uniqueness of states is centred around that region of parameter space in which the flow allows convergence towards the plane of symmetry. As pointed out by a referee, one might therefore associate the appearance of alternative states with an ability to tune the far-field flow in such a manner that self-similarity is preserved.

On approaching $\Psi_{\infty}=2$, it becomes increasingly difficult to locate the exponential states using either of our numerical techniques. The exponential modes (which only exist for $\Psi_{\infty}>0$ ) are smoothly embedded in the continua of algebraic modes, and it becomes increasingly difficult to distinguish numerically between those states with $A_{3}=0$ (in expression (2.10)) and the neighbouring algebraically, but nevertheless rapidly decaying states.

For $\Psi_{\infty} \in(0,2)$, there is a continuum of states that, in general, decay algebraically although with a discrete set of exponential states embedded within the continuum. For $\Psi_{\infty}=2$ the decay is always exponential rather than algebraic in the far field; however a continuum of solutions still remains. In general, the cross-flow component $\Psi(\eta)$ has an algebraic component of the form $\eta^{-2 \Psi_{\infty} /\left(2-\Psi_{\infty}\right)}$, which becomes exponential in the limit of $\Psi_{\infty} \rightarrow 2$. In figure 2 we show profiles of $\Psi(\eta)$ and $\Phi(\eta)$ from the continuum of exponentially decaying states at $\Psi_{\infty}=2$; by contrast, profiles of the streamwise component $U(\eta)$ vary only slightly through the continuum of states. We note that this continuum of solutions to the three-dimensional stagnation-point flow over a plane boundary has been previously discussed in the work of Hall et al. (1992). 

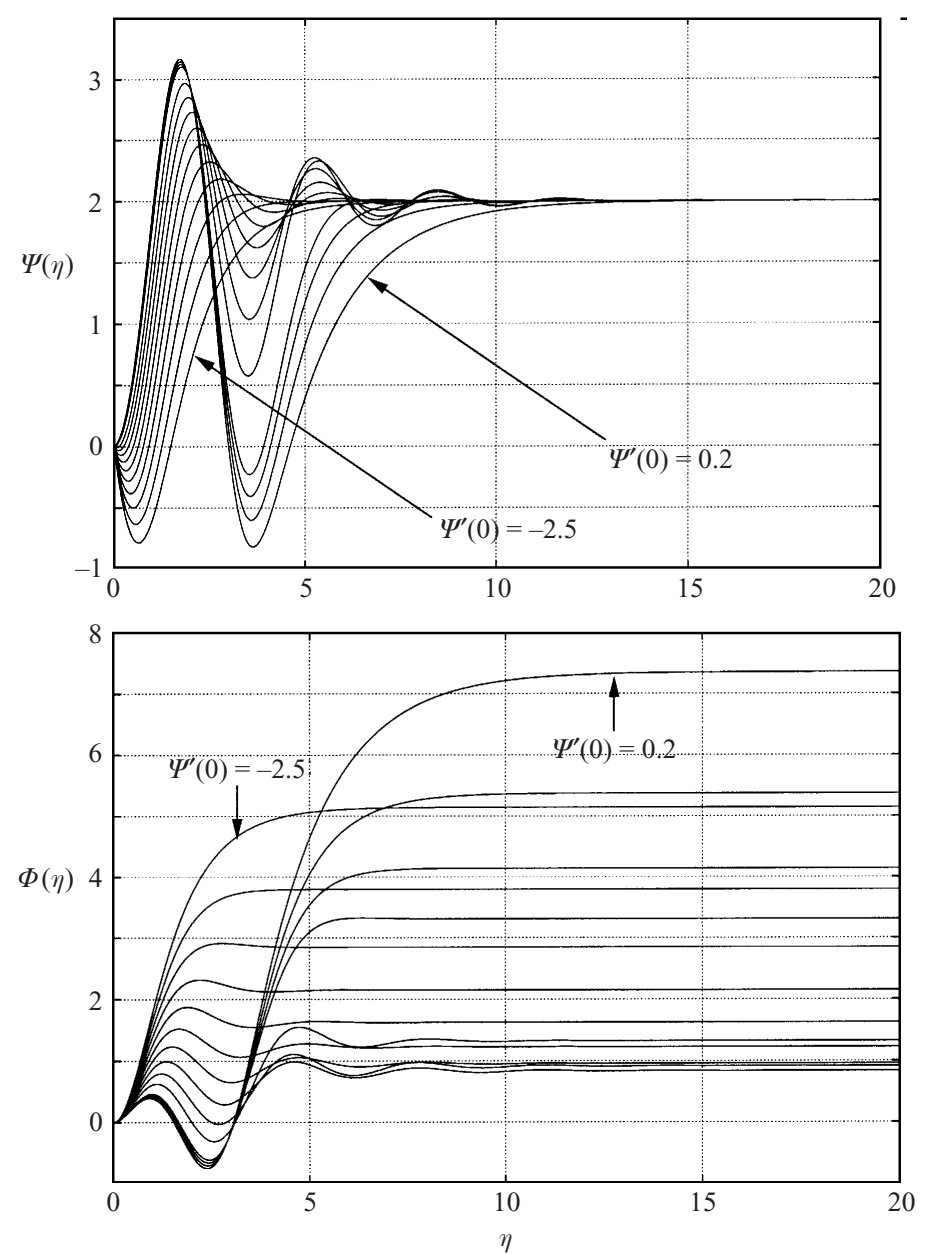

Figure 2. Profiles of $\Psi(\eta)$ and $\Phi(\eta)$ for the continuum of states at $\Psi_{\infty}=2$ (with $n=1$ ). Profiles of $U$ all decay rapidly towards the edge value $(U \rightarrow 1)$ and vary only slightly throughout the continuum. Profiles of states in the continuum are shown parameterized by $\Psi^{\prime}(0)=\{0.2,0.15,0.05\},\{-0.25,-0.5, \ldots,-2.5\}$.

\subsection{The general solution structure for $n \in[0,1)$ : exponential modes}

For $n \neq 1$ the solutions are no longer exact and rely on a boundary-layer approximation. In figure 3 we present details of the exponential modes for $n=0$. The point identified by the filled circle in figure $3(a)$ denotes the Blasius $(n=0)$ solution (with $U^{\prime}(0) \approx 0.4696$ ), which is two-dimensional with no cross-flow, corresponding to $U(\eta) \equiv \Psi(\eta)$ and $\Psi_{\infty}=1$. The solution identified by the open circle is an alternative to the Blasius solution, which differs in that it is three-dimensional, although since $\Psi_{\infty}=1-n=1$, the external flow nevertheless remains two-dimensional; this mode is described in Duck et al. (2000).

Comparison of the results of figures 1 and 3 shows a qualitative difference in that the solution structures near $\Psi_{\infty}= \pm 2$ are disconnected when $n=0$. There is a critical value of $n \approx 0.25$ at which the exponential states of branches 1 and 2 disconnect through a transcritical bifurcation. In figure $3(b)$ we show the locus of the two saddle-node points in the $\left\{\Psi_{\infty}, n\right\}$; note that figure $3(b)$ is presented such that 

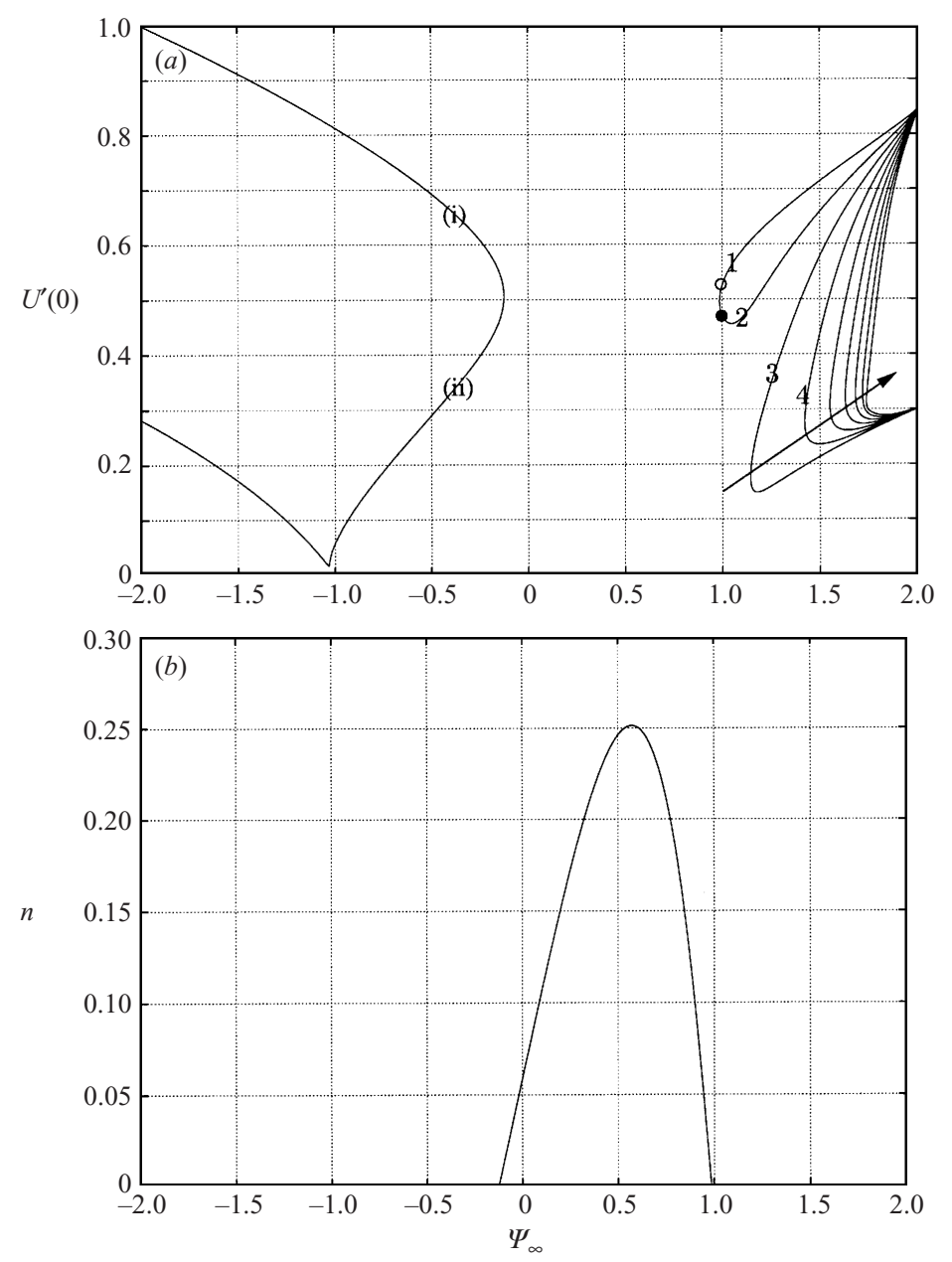

FiguRE 3. (a) Properties of the 'exponential modes' for varying external cross-flow, $\Psi_{\infty}$ and $n=0$. The filled circle denotes the two-dimensional Blasius solution, for which $U(\eta)=\Psi(\eta)$ in our formulation. The open circle denotes a three-dimensional alternative to the Blasius state that includes a jet-like cross-flow component, although no cross-flow exists in the free stream. $(b)$ As $n$ is increased, the two saddle points where branches (i) and (ii) and (1) and (2) connect (as shown in $(a)$ above) approach each other until recombining through a transcritical bifurcation at $n \approx 0.25$.

the locations of the two resulting saddle-node points for $n=0$ are aligned with the corresponding data in figure $3(a)$.

The cascade of exponential modes observed near $\Psi_{\infty}=2$ persists for $n \neq 1$ and again we show just a subset of the infinite sequence of states. In $\S 4$ we shall show that this cascade of states can be described asymptotically.

We have already noted that the displacement thickness, $\delta^{*}$, plays an important role in the asymptotic analysis. In figure 4 we show the behaviour of $\delta^{*}$ for the exponential modes in the two cases $n=0$ and $n=1$. From both these figures it appears that the dual limit of $\Psi_{\infty} \rightarrow 2$ and $\delta^{*} \rightarrow 4$ is of some significance, and this issue will be taken up later in the paper. 

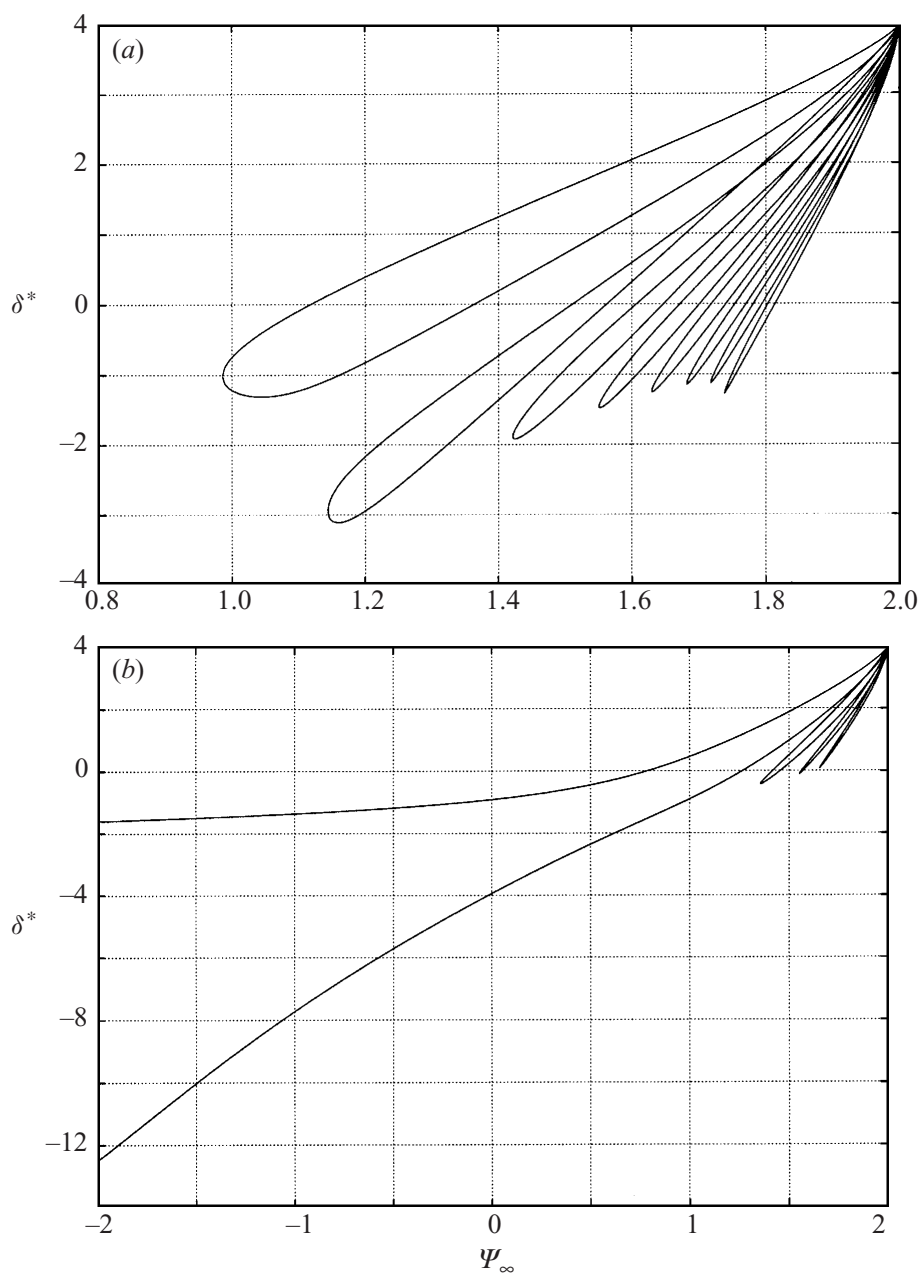

FIGURE 4. Properties of the 'exponential modes' for varying cross-flow, $\Psi_{\infty}$, with $n=0(a)$ and $n=1(b)$.

\subsection{Algebraically decaying boundary-layer states}

For $\Psi_{\infty}>0$ the exponential modes described above form only a discrete subset of the full solution set. For $n>0$, there is a continuum of states that have an algebraic behaviour in the far field of $\Psi(\eta)$. Furthermore, at a fixed value of the cross-flow parameter, in general there may be several continua of states. As an example, in figure 5 we show two continua in the case $n=0$ at fixed $\Psi_{\infty}=1.73$ and $\Psi_{\infty}=1.80$. We see two continua of states in the plane spanned by $U^{\prime}(0)$ and $\delta^{*}$; therefore $\Psi^{\prime}(0)$ varies along these lines, whilst the exponential solutions are located at discrete points along these lines. For $\Psi_{\infty}>\Psi_{t c}$ (as shown in figure $5 a$ ) there are two distinct continua of states that remain disconnected. However, at $\Psi_{\infty}=\Psi_{t c}$ a transcritical bifurcation reconnects the two continua in the usual manner.

For $\Psi_{\infty}>\Psi_{t c}$ there is a closed region in the parameter plane for which no solutions can be located. The locus of the saddle-node points arising from the transcritical bifurcation is shown in figure 6 in the plane spanned by $\left\{U^{\prime}(0), \Psi_{\infty}\right\}$. The continua of states all terminate at critical points where the solution is singular with $\delta^{*} \rightarrow \pm \infty$. 

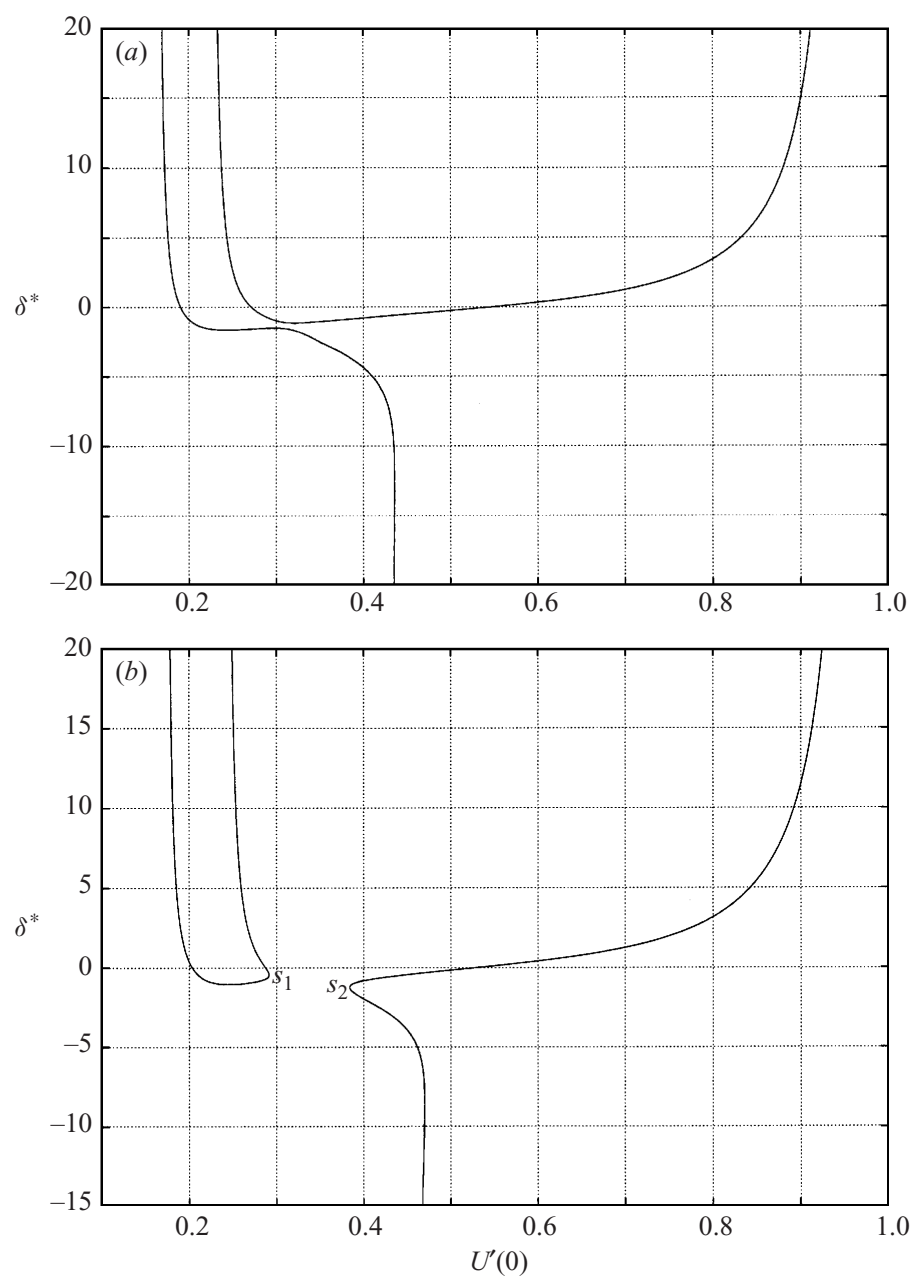

FIGURE 5. (a) The two continua of boundary-layer states for $\Psi_{\infty}=1.73<\Psi_{t c}$ and $n=0$. (b) The same two continua of states for $\Psi_{\infty}=1.8>\Psi_{t c}$ and $n=0$ (where $\Psi_{t c}$ denotes the location of a transcritical bifurcation). The two saddle-node points $s_{1,2}$ separate for increasing $\Psi_{\infty}$, the exact behaviour of which is shown in figure 6 .

The structure of the velocity profiles and the corresponding asymptotic description of the flow in these limits is considered briefly in $\S 5$.

\section{A cascade of exponential modes}

It is evident from the results presented in figures 1-6 that any asymptotic description of the role played by the exponential modes must be centred around the critical value of $\Psi_{\infty}=2$. In fact, one may conjecture, based on the numerical evidence, that there is a cascade of exponential modes with an infinity of such states occurring as $\Psi_{\infty} \rightarrow 2^{-}$. The physical interpretation of $\Psi_{\infty}=2$ is a boundary-layer flow for which the crossflow component is equal in magnitude to the streamwise velocity in the external flow, but having opposite sign, with the flow directed towards the plane of symmetry $z=0$; as such this may be interpreted as a boundary-layer collision process. In the following analysis any (positive) value of the parameter $n$ is permitted. 


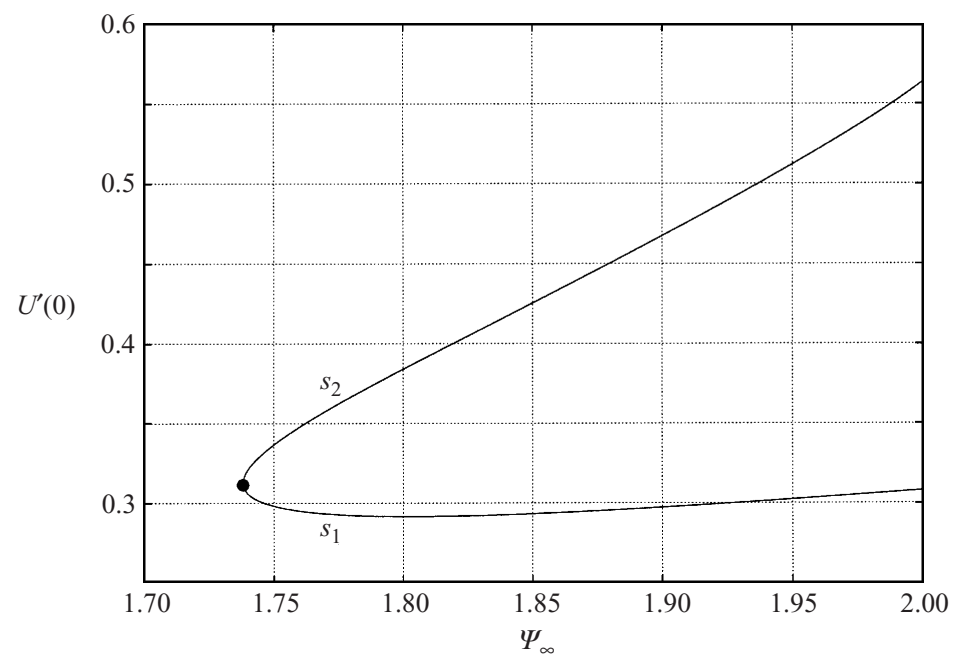

FIGURE 6. The transcritical bifurcation (denoted by the filled circle) at $\Psi_{\infty}=\Psi_{t c} \approx 1.738$, and the locus of the resulting saddle-node points $s_{1,2}$ (as shown in figure 5) for $\Psi_{\infty}>\Psi_{t c}(n=0)$.

4.1. The cascade of exponential modes as $\Psi_{\infty} \rightarrow 2^{-}$for general $\delta^{*}$

We now seek a description of the far-field structure of $\Psi(\eta)$ for

$$
\Psi_{\infty}=2-\varepsilon,
$$

where $0<\varepsilon \ll 1$. Prompted by the results of our numerical investigation we shall concentrate on the far-field behaviour of the cross-flow velocity component $\Psi(\eta)$ (the streamwise velocity component $U(\eta)$ plays a minor role in this description), utilizing in particular (2.7).

From (2.8) (for $\eta \gg 1$ ) we obtain

$$
\psi^{\prime \prime}+\left(\varepsilon \eta+\delta^{*}\right) \psi^{\prime}+(4-2 \varepsilon) \psi=0
$$

which, for $\varepsilon \eta \ll 1$, leads to

$$
\psi(\eta)=A_{1} \exp \left(-\delta^{*} \eta / 2\right) \sin \left(\frac{\mu \eta}{2}+A_{2}\right)+A_{3} \eta^{-4 / \varepsilon}+\cdots
$$

where $\mu=\sqrt{16-\delta^{* 2}}$ (which we shall take to be $O(1)$ ) and $A_{1,2,3}$ are real constants. There is a clear indication from the numerical results that $\delta^{*} \leqslant 4$; see figure 4 . As far as the asymptotic description is concerned, $A_{1,2}$ are known constants, since they can be determined numerically from the solution at $\Psi_{\infty}=2$ for any given $\delta^{*}$. Furthermore, since we wish to describe the cascade of exponential modes we shall take $A_{3}=0$, thereby eliminating the algebraic behaviour in the far field with a concomitant loss of any continua of solutions.

The aim is to show that a discrete set of exponential states can be captured in the perturbation expansion above. It is helpful to first consider an outer region by the introduction of a scaled coordinate in the form

$$
\xi=\varepsilon \eta+\delta^{*},
$$

together with the substitution

$$
\psi=F(\xi) \exp \left(-\xi^{2} / 4 \varepsilon\right),
$$




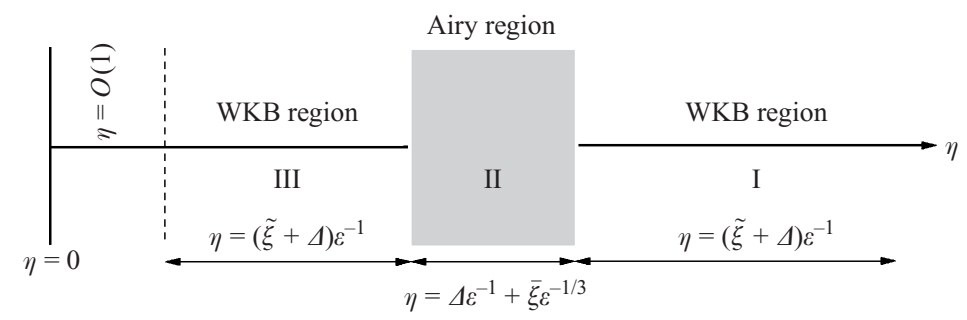

FIgURE 7. Asymptotic regions as $\Psi_{\infty} \rightarrow 2^{-}$, with $0<4-\delta^{*}=\Delta=O(1)$ With regions I, II, III spanned by the scaled coordinates $\tilde{\xi}, \bar{\xi}=O(1)$.

for which (4.2) leads to the following problem

$$
\varepsilon^{2} F^{\prime \prime}(\xi)=\left(\frac{\xi^{2}}{4}-4+\frac{5 \varepsilon}{2}\right) F(\xi)
$$

which we note is of parabolic cylinder form, albeit with asymptotically large order.

It is perhaps worth commenting that systems of this type are obtained when considering model problems in quantum mechanics, and can be viewed as essentially a Schrödinger equation for energy eigenstates in a weakly varying potential. In this case the axial velocity profile is related to the 'potential-well' function, and the 'energy levels' correspond to the displacement thickness $\delta^{*}$, although here this dependence has been concealed in the definition of $\xi$. We approach the solution of (4.6) via a WKB method and may anticipate that a new region will be required at a critical location for which $\xi^{2} / 4-4=0$; that is, as $\xi \rightarrow 4$. It is therefore convenient to introduce a translated coordinate $\tilde{\xi}=\xi-4$, and then to define

$$
G_{0}(\tilde{\xi})=\frac{\tilde{\xi}^{2}+8 \tilde{\xi}}{4},
$$

which is the leading-order form of the bracketed term on the right of (4.6).

We shall describe the outer solution in three regions, as illustrated in figure 7.

Region I: An outer WKB region with $0<\tilde{\xi}=O(1)$

In this outermost region, having eliminated the unbounded solution as $\tilde{\xi} \rightarrow \infty$, we obtain a WKB solution of $F=F_{I}(\tilde{\xi})$, the leading-order form of which is (following the usual WKB procedure)

$$
F_{I}(\tilde{\xi})=\frac{C_{I}}{\left[G_{0}(\tilde{\xi})\right]^{1 / 4}} \exp \left(-\frac{1}{\varepsilon} \int_{0}^{\tilde{\xi}} \sqrt{G_{0}(s)} \mathrm{d} s\right),
$$

where the amplitude constant $C_{I}$ is to be determined.

Region II: An Airy region with $\bar{\xi}=\varepsilon^{-2 / 3} \tilde{\xi}=O(1)$.

In the usual manner for such problems, one can see that the WKB solution above becomes invalid for $\left|G_{0}(\tilde{\xi})\right|$ sufficiently small. We therefore obtain a new region spanned by the rescaled coordinate $\bar{\xi}=\varepsilon^{-2 / 3} \tilde{\xi}=O(1)$, which corresponds to a layer of width $O\left(\varepsilon^{-1 / 3}\right)$ centred at a critical location $\eta=\left(4-\delta^{*}\right) \varepsilon^{-1}$ in terms of the original transverse boundary-layer coordinate.

In this layer $G_{0} \sim 2 \varepsilon^{2 / 3} \bar{\xi}+\cdots$, and therefore the leading-order form for $F$ in region 
II is given by

$$
F_{I I}=C_{I I} \operatorname{Ai}\left(2^{1 / 3} \bar{\xi}\right)
$$

where $C_{I I}$ is a further amplitude constant to be determined.

We note here that the second solution $\operatorname{Bi}\left(2^{1 / 3} \bar{\xi}\right)$ has been removed by matching with the exponentially decaying behaviour in region II above as $\bar{\xi} \rightarrow \infty$.

Region III: An outer WKB region with $0<-\tilde{\xi}=O(1)$

In this second WKB region we must retain both exponential solutions, which are more conveniently written in terms of a circular function. Thus, in this region we obtain a leading-order solution in the form

$$
F_{I I I}=\frac{C_{I I I}}{\left[-G_{0}(\tilde{\xi})\right]^{1 / 4}} \sin \left(\frac{1}{\varepsilon} \int_{0}^{\tilde{\xi}} \sqrt{-G_{0}(s)} \mathrm{d} s+D_{I I I}\right),
$$

where $C_{I I I}$ is an amplitude constant and $D_{I I I}$ is a phase constant, both of which must be determined.

A straightforward matching process allows us to determine the relationships between the amplitude constants $C_{I}, C_{I I}$ and $C_{I I I}$ however we do not provide explicit formulae here. Of more significance is the process of matching the phase constants in regions II, III with the corresponding phase $A_{2}$ of expression (4.3).

We begin by noting that, for large negative argument,

$$
\operatorname{Ai}(s) \sim \frac{1}{\sqrt{\pi}}(-s)^{1 / 4} \sin \left(\frac{2}{3}(-s)^{3 / 2}+\frac{\pi}{4}\right),
$$

and therefore matching the phase of expressions (4.9) and (4.10) reveals that $D_{I I I}=$ $\pi / 4$.

Similarly, we must match the phase of $F_{I I I}$ above with that in (4.3), which leads to

$$
\left(A_{2}+m \pi\right)=\frac{\pi}{4}+\frac{1}{\varepsilon} \int_{0}^{-\Delta} \sqrt{-G_{0}(s)} \mathrm{d} s,
$$

for any integer $m$. This upper limit of the integral arises from the fact that approaching the region $\eta=O(1)$ corresponds to $\tilde{\xi} \rightarrow-\Delta=\delta^{*}-4$ in this region.

This connection formula can be given more explicitly by evaluating the integral, which leads to

$$
\frac{\mu \delta^{*}}{4}+4 \arcsin \left(\frac{\delta^{*}}{4}\right)-2 \pi+\varepsilon P\left(\delta^{*}\right)+O\left(\varepsilon^{2}\right)=\varepsilon\left(A_{2}\left(\delta^{*}\right)+m \pi-\frac{\pi}{4}\right) .
$$

Here we note that, formally, there is an $O(\varepsilon)$ term, $P\left(\delta^{*}\right)$, which arises from the previously neglected $O(\varepsilon)$ term present in (4.6). However, a balance of (4.13) can only be achieved for $m=O\left(\varepsilon^{-1}\right)$ or $\delta^{*} \approx 4$, and in both cases it can be shown that $P\left(\delta^{*}\right)$ plays no role. Thus, (4.13) provides a functional relationship between $\delta^{*}$ and $\varepsilon$. For a fixed $\delta^{*}$ there is a sequence of discrete $\varepsilon=\varepsilon_{m}$, for $|m|$ sufficiently large, at which the far-field behaviour of $\Psi(\eta)$ contains no algebraic component.

In terms of figure 4, we may interpret (4.13) as: (i) for a fixed $m$, exponentially decaying modes must have $\delta^{*} \rightarrow 4$ as $\Psi_{\infty} \rightarrow 2^{-}$(as shown in the figure), and (ii) for a fixed $\delta^{*}$, exponential modes can be found at $\Psi_{\infty}=2-\varepsilon(0<\varepsilon \ll 1)$ only for sufficiently large $m=O\left(\varepsilon^{-1}\right)$. The phase-shift constant $|m|=1,2,3, \ldots$, parameterizes the discrete solution set in the neighbourhood of $\Psi_{\infty}=2$. Therefore, only the branches of asymptotically high order can be found near $\Psi_{\infty}=2$ at a general $\delta^{*}$, whereas all 


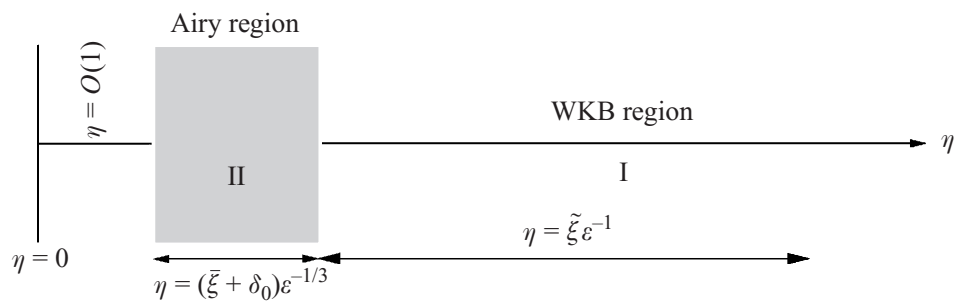

FIGURE 8 . Asymptotic regions as $\Psi_{\infty} \rightarrow 2^{-}$, with $\delta^{*}=4-\delta_{0} \varepsilon^{2 / 3}$. With regions I, II spanned by the scaled coordinates $\tilde{\xi}, \bar{\xi}=O(1)$.

branches can be found near $\delta^{*}=4$ in agreement with figure 4 . Additionally, we note that the number of zeros of $\Psi-1$ increases in proportion to $1 /\left(2-\Psi_{\infty}\right)$ as $\Psi_{\infty} \rightarrow 2^{-}$.

The solutions for fixed ' $m$ ', with $\delta^{*} \rightarrow 4^{-}$

For $m$ fixed one must seek a solution to (4.13) in the form $\delta^{*}=4-\tilde{\delta}(\varepsilon)$. As $\delta^{*} \rightarrow 4^{-}$, $A_{2}=o(1)$ and it is clear from the limiting form of (4.13) that a non-trivial balance can only be achieved for $\tilde{\delta}(\varepsilon)=\delta_{0} \varepsilon^{2 / 3}$, where $\delta_{0}$ satisfies

$$
\delta_{0}=\left[\frac{3 \pi}{2 \sqrt{2}}\left(m-\frac{1}{4}\right)\right]^{2 / 3} .
$$

As we shall see below, this is the high-mode-number limit of an asymptotic expansion about the accumulation point of $\Psi_{\infty}=2, \delta^{*}=4$ shown clearly in figure 4 .

4.2. A description of the accumulation point; $\Psi_{\infty}=2-\varepsilon$ and $\delta^{*}=4-\delta_{0} \varepsilon^{2 / 3}+\cdots$

The expression (4.13) only provides a solution away from $\delta^{*}=4$. For a description valid at the accumulation point we must take into account that the Airy region (previously found at a critical position in the outer WKB solution) approaches the innermost layer, as shown in figure 8. Furthermore, rather than (4.3), we have a leading-order profile for $\psi$ that exists without spatial oscillations since $\mu \rightarrow 0$ as $\delta^{*} \rightarrow 4$.

In region I, the solution proceeds as before, with just the exponentially decaying term present in the WKB expansion. The solution in the Airy region (II) is again given by (4.9), which can be rewritten in terms of the unscaled coordinate, $\eta$, as

$$
F_{I I}=C_{I I} \operatorname{Ai}\left[2^{1 / 3}\left(\varepsilon^{1 / 3} \eta-\delta_{0}\right)\right] .
$$

This solution must be matched directly to the solution for $\eta \gg 1$, $\varepsilon \eta \ll 1$, which in this case leads to

$$
\psi(\eta)=\left(A_{I}+A_{I I} \eta\right) \mathrm{e}^{-2 \eta}+A_{I I I} \eta^{-4 / \varepsilon}+\cdots
$$

rather than (4.3), since $\delta^{*} \approx 4$.

For a solution with no algebraic component we take $A_{I I I}=0$, and to match with the solution in the Airy region we require that

$$
\delta_{0}=-2^{-1 / 3} \alpha_{j}, \quad j=1,2, \ldots,
$$

where $\alpha_{j}$ is the $j$ th zero of the Airy function. Clearly, for large $j$ (with $\delta_{0} \rightarrow \infty$ ) we can use the asymptotic form for the high-order zeros of the Airy function, as determined from (4.11), to recover the expression given for the outer region as (4.14) above.

In summary, we predict an infinite (discrete) sequence of exponentially decaying solutions that differ only in the far field and are most easily distinguished through 

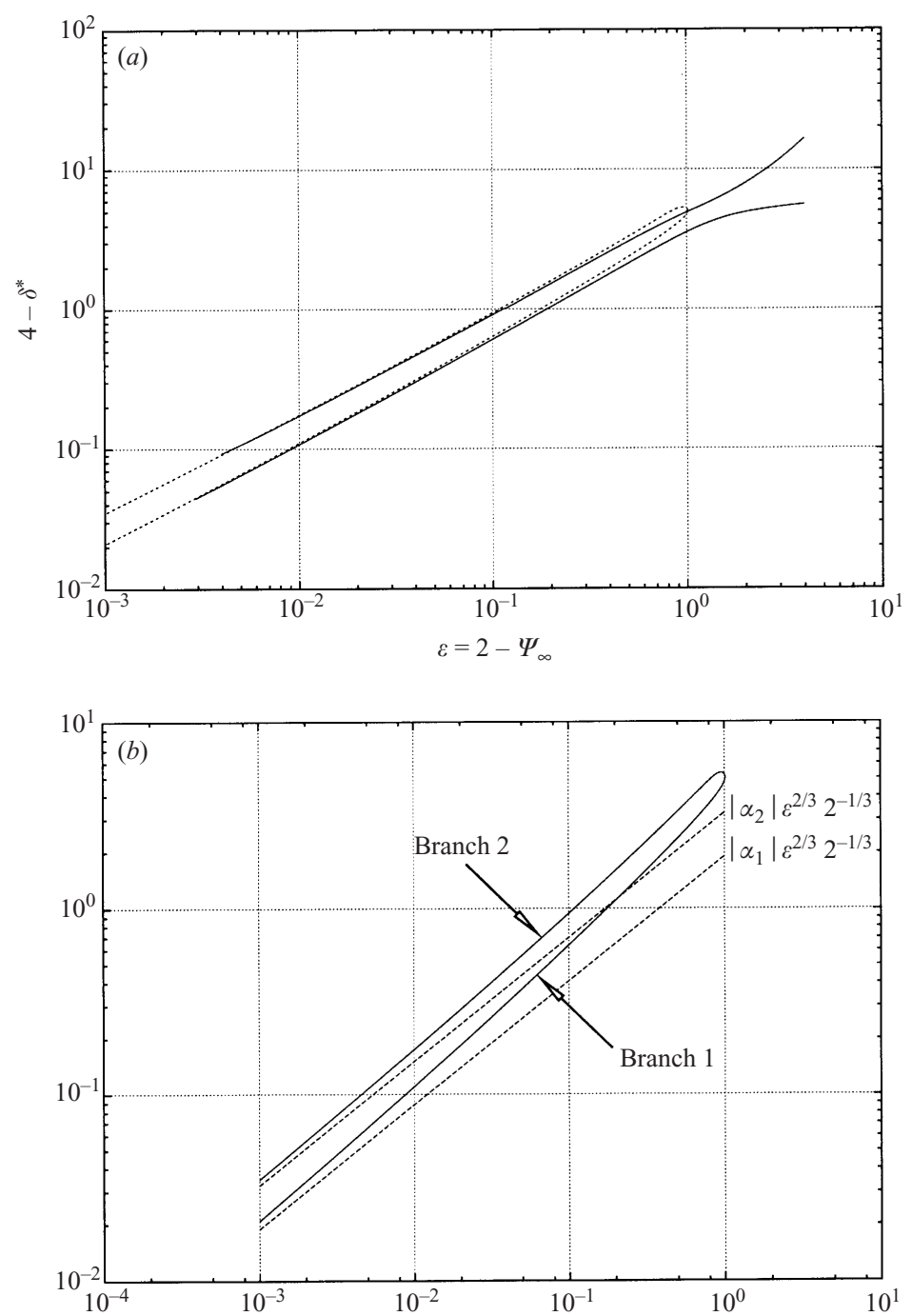

Figure 9. (a) A comparison between the approach to $\delta^{*}=4$ for $n=0$ (dotted line) and $n=1$ (solid line), showing the same behaviour in both cases for the first two modes. (b) A quantitative comparison of numerical data for $n=0$ with the leading-order asymptotic prediction for the first two modes, where $\operatorname{Ai}\left(\alpha_{j}\right)=0$.

the corresponding values of the displacement thickness. This latter quantity for the $j$ th exponential mode is

$$
\delta^{*}=4+2^{-1 / 3} \alpha_{j} \varepsilon^{2 / 3}+O(\varepsilon), \quad \text { for } \quad j=1,2,3, \ldots,
$$

for $\Psi_{\infty}=2-\varepsilon$. We note that this cascade of states only requires $n \geqslant 0$ and is a generic property, being otherwise independent of the Falkner-Skan parameter, $n$, to this order.

A comparison of numerical results in the neighbourhood of $\Psi_{\infty}=2$ and $\delta^{*}=4$ is given in figures $9(a)$ and $9(b)$. Figure $9(a)$ confirms the prediction that the asymptotic approach to the accumulation point is in fact independent of $n$, and shows the first two exponential modes for the cases of $n=0$ and $n=1$. Figure $9(b)$ shows 
a quantitative comparison of the data for $n=0$ with the predicted leading-order asymptotic approach, taken with $\alpha_{1} \approx-2.338$ and $\alpha_{2} \approx-4.088$ being the first two zeros of the Airy function.

\section{Other asymptotic limits: $\delta^{*} \rightarrow \pm \infty$}

As can be observed from the numerical results described in $\$ 3$ (see figure 5), in general, continua of states exist between limiting points at which $\left|\delta^{*}\right| \rightarrow \infty$. Here we describe briefly the asymptotic solution in the neighbourhood of these singular points.

\subsection{The limit $\delta^{*} \rightarrow-\infty$}

The results of figure 5 show that (for $\Psi_{\infty} \neq 2$ ) the continua of algebraic states may have a limiting behaviour in which $\delta^{*} \rightarrow-\infty$.

In the case $n=0$ it is simplest to consider the alternative formulation of (2.3) as an initial-value problem using the symmetry (2.5). Starting from the initial conditions given by (2.6), we may examine the evolution for any fixed constant $K$, over a range of $\Lambda=\Psi^{\prime}(0)$. A corresponding sample of velocity profiles is shown in figure 10 , in which the presence of a growing lengthscale is clearly indicated as well as an outer transition layer present in regions of otherwise uniform behaviour.

As can be seen from figure 10, the velocity profiles in this limit are extremely oscillatory and we anticipate these to be of little physical importance. For the sake of completeness we provide a brief overview of this solution structure asymptotically, by considering a perturbation of $\Lambda$ about the critical value $\left(\Lambda^{*}\right)$ at which $\delta^{*} \rightarrow-\infty$, by writing $\Lambda=\Lambda^{*}-\varepsilon$, where $0<\varepsilon \ll 1$.

The inner-layer solution: $\eta=O(1)$

We seek a solution over an $O(1)$ lengthscale (for a fixed value of $K$ ) such that

$$
U_{0} \rightarrow U_{e}, \quad \Psi_{0} \rightarrow \Psi_{e} \quad \text { as } \quad \eta \rightarrow \infty,
$$

in accordance with the numerical results, where $U_{0}$ and $\Psi_{0}$ are the leading-order terms in expansions of $U$ and $\Psi$ respectively. Given these conditions, correspondingly we assume $\Phi_{0} \rightarrow \Phi_{e}$. Here $U_{e}, \Phi_{e}$ and $\Psi_{e}$ are constants, which for a fixed $K$, on account of (2.3) with $n=0$, must satisfy

$$
2 U_{e}-\Psi_{e}=0, \quad \Psi_{e}^{2}-U_{e}^{2}=K,
$$

from the form of continuity (since $\Phi_{e}$ is a constant) and the leading-order far-field balance of the streamwise momentum equation.

We therefore obtain

$$
U_{e}=\sqrt{\frac{K}{3}}, \quad \Psi_{e}=2 \sqrt{\frac{K}{3}},
$$

and this provides a numerical problem that determines the critical value $\Lambda^{*}=\Lambda^{*}(K)$, since for a given $K$ one must choose $\Lambda^{*}$ such that (5.3) is satisfied. Hence we proceed by assuming that $\Lambda^{*}$ is known. Furthermore, we note that the numerical evidence consistently indicates that $U_{e}, \Psi_{e}>0$, although we have not attempted to prove that this is the only possibility within the asymptotic framework. For the case depicted in figure 10 we can obtain $U_{e} \approx 1.4$ and $\Psi_{e} \approx 2.8$, in agreement with the numerical results. 

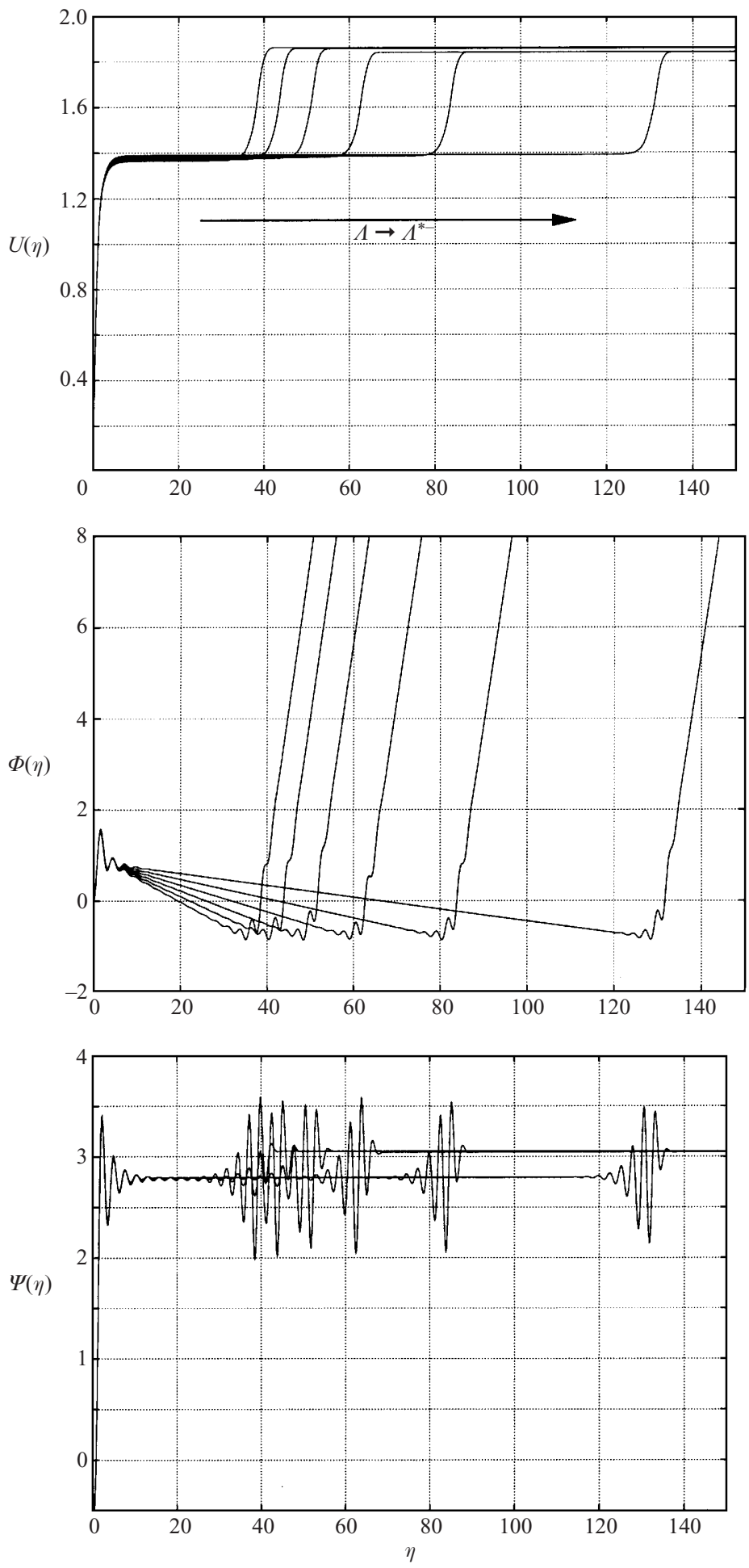

FIGURE 10 . Profiles of the velocity components in the limit of $\delta^{*} \rightarrow \infty$ with $n=0$ and $K \approx 5.88$ (presented for six increasing values of $\Lambda \approx-2.3977,-2.3877, \ldots,-2.3477) ; \Psi^{\prime}(0)=\Lambda^{*}$ is the critical value at which $\delta^{*} \rightarrow \infty$ in the initial-value formulation of (2.6). Note the existence of an outer transition layer that separates two regions of uniform $U$ and $\Psi$. 
We now consider the far-field behaviour of these leading-order solutions by introducing

$$
U_{0}=U_{e}+u, \quad \Phi_{0}=\Phi_{e}+\phi, \quad \Psi_{0}=\Psi_{e}+\psi
$$

where $|u|,|\phi|,|\psi| \ll 1$ and $|u| \ll|\psi|$. Furthermore, it is easily observed that the far-field behaviour of the next-order term for $\Phi$ is

$$
\Phi_{1}=\delta_{1}+\varepsilon \gamma \eta,
$$

where $\delta_{1}$ and $\gamma$ are constants that can be determined.

In the particular case of $n=0$, the governing equations in the far field are therefore

$$
\begin{aligned}
u^{\prime \prime}+\left(\Phi_{e}+\gamma \eta \varepsilon\right) u^{\prime} & =0, \\
\psi^{\prime \prime}+\left(\Phi_{e}+\gamma \eta \varepsilon\right) \psi^{\prime}+2 \Psi_{e} \psi & =0 .
\end{aligned}
$$

The outer layer: $\hat{\eta}=\varepsilon \eta=O(1)$

In an outer region, (5.6a) can be approached again using a WKB expansion, with

$$
\psi=A \exp \left(\frac{1}{\varepsilon} \sum_{n=0}^{\infty} K_{n}(\hat{\eta}) \varepsilon^{n}\right),
$$

where $A$ is a constant, and it can be shown that

$$
K_{0}(\hat{\eta})=-\frac{\Phi_{e} \hat{\eta}}{2}-\frac{\gamma \hat{\eta}^{2}}{4} \pm i \sqrt{2 \Psi_{e}} \int^{\eta}\left(1-\frac{\left(\Phi_{e}+\gamma s\right)^{2}}{8 \Psi_{e}}\right)^{1 / 2} \mathrm{~d} s .
$$

Here, $1-\left(\Phi_{e}+\gamma s\right)^{2} / 8 \Psi_{e} \geqslant 0$ in our region of interest and clearly there is exponential growth beyond the critical location $\eta=\hat{\eta}_{0} / \varepsilon$, where

$$
\hat{\eta}_{0}=-\frac{2 \Phi_{e}}{\gamma} .
$$

At the critical location, the axial flow is

$$
\Phi=\Phi_{e}+\gamma \hat{\eta}_{0}=-\Phi_{e}
$$

Both the location of the transition region $\left(\eta=\hat{\eta}_{0} / \varepsilon\right)$ and the predicted axial velocity $-\Phi_{e}$ have been verified against the full numerical solutions in the limit of $\Lambda \rightarrow \Lambda^{*}$. Clearly, for $\eta>\hat{\eta}_{0} / \varepsilon$ there is an oscillatory exponential growth, and a further region is required in which all quantities balance. This outer transition region is of $O(1)$ width, in which the governing equations are those of the fully nonlinear system (2.3). Although described above for the particular case of $n=0$, the same general solution structure applies for any $n \geqslant 0$.

\subsection{The limit $\delta^{*} \rightarrow \infty$}

There is a further asymptotic limit in which $\delta^{*} \rightarrow \infty$. In this case the $O(1)$ innerlayer solution is simply the corresponding exponentially decaying solution obtained at $-\Psi_{\infty}$, that is, one applies the edge condition $\Psi \rightarrow-\Psi_{\infty}$ to the inner-layer solution. The match to the correct far-field behaviour of $\Psi \rightarrow \Psi_{\infty}$ is then achieved through an outer region. The outer region is of width $O\left(\delta^{*}\right)$, with $U, \Psi=O(1)$ and $\Phi=O\left(\delta^{*}\right)$, in which the leading-order equations correspond to the full governing system but without the viscous terms. 


\section{Stability issues}

The unusual (continuous) multiplicity of states raises the natural question of which of these states are physically realizable. As noted earlier, Davey \& Schofield (1967) and Schofield \& Davey (1967) made the somewhat arbitrary decision to select the solution with the fastest (exponential) decay into the free stream as being the physically most important; here we attempt to make the decision on more rational grounds. The natural way to investigate this issue is to perform stability analyses of the various states, and this is the procedure to be followed here, by investigating two classes of disturbance. The first corresponds to a three-dimensional, steady class, which grows algebraically in the streamwise $(x)$ direction, whilst retaining the same dependence as the base flow in the spanwise $(z)$ direction; see (2.1). This type of disturbance has been investigated for the case of Falkner-Skan and other, related, three-dimensional boundary layers (albeit with no free-stream cross-flow velocity components) by Duck \& Dry (2001) and Duck (2002). From a mathematical point of view, the attractive feature of this method (which is also related the work of Luchini (1996), is that the procedure is entirely rational within the context of the boundary-layer approximation. Additionally, the occurrence of such instability modes has been linked to the 'by-pass' transition process by Kachanov (1994), for example, and as such these are likely to be of considerable practical importance. The second type of imposed disturbance is a purely temporal one. The important feature of this class is that for $n=1$ the procedure is exact within the framework of the Navier-Stokes equations.

There are a number of other candidates for possible instabilities in the flows under consideration. Cross-flow instabilities (see for example Malik (1986) are an obvious possibility, as are Tollmien-Schlichting instabilities (see Drazin \& Reid 1981, for example); however, these (and other) forms require additional approximations and analyses. We therefore restrict our attention to the two types of disturbance outlined above, both of which rely broadly on the type of analysis that has been the main thrust of this paper.

\subsection{Steady disturbances growing in the streamwise direction}

In this case we seek steady three-dimensional disturbances which take on the same spanwise form as the base state. For our purposes, this is best achieved by taking (2.1) again, but making the following changes for the dependent variables:

$$
U \rightarrow U(\eta)+x^{\lambda} \tilde{u}(\eta)+\cdots, \quad \Phi \rightarrow \Phi(\eta)+x^{\lambda} \tilde{\phi}(\eta)+\cdots, \quad \Psi \rightarrow \Psi(\eta)+x^{\lambda} \tilde{\psi}(\eta)+\cdots
$$

Here, we take $x \ll 1$, where $\lambda$ is an eigenvalue (at least in the first instance), to be determined. The expansions (6.1) are then substituted into the full (steady) three-dimensional boundary-layer equations, and this leads to

$$
\begin{gathered}
2 \tilde{u}+2 \lambda \tilde{u}=\tilde{\phi}_{\eta}+\tilde{\psi}, \\
\tilde{u}_{\eta \eta}=4 n U \tilde{u}+2 \lambda U \tilde{u}-\tilde{\phi} U_{\eta}-\tilde{u}_{\eta} \Phi, \\
\tilde{\psi}_{\eta \eta}-2\left(1-n^{2}\right) U \tilde{u}+\Phi \tilde{\psi}_{\eta}+\tilde{\phi} \Psi_{\eta}+2 \Psi \tilde{\psi}-2 \lambda U \tilde{\psi}=0,
\end{gathered}
$$

subject to

$$
\tilde{u}(0)=\tilde{\phi}(0)=\tilde{\psi}(0)=0,
$$

and

$$
\tilde{u}, \tilde{\psi} \rightarrow 0 \text { as } \eta \rightarrow \infty .
$$

Note that a similar approach was adopted by Duck \& Dry (2001). The system (6.2) was solved using a combination of direct (QZ methods, in conjunction with finite- 


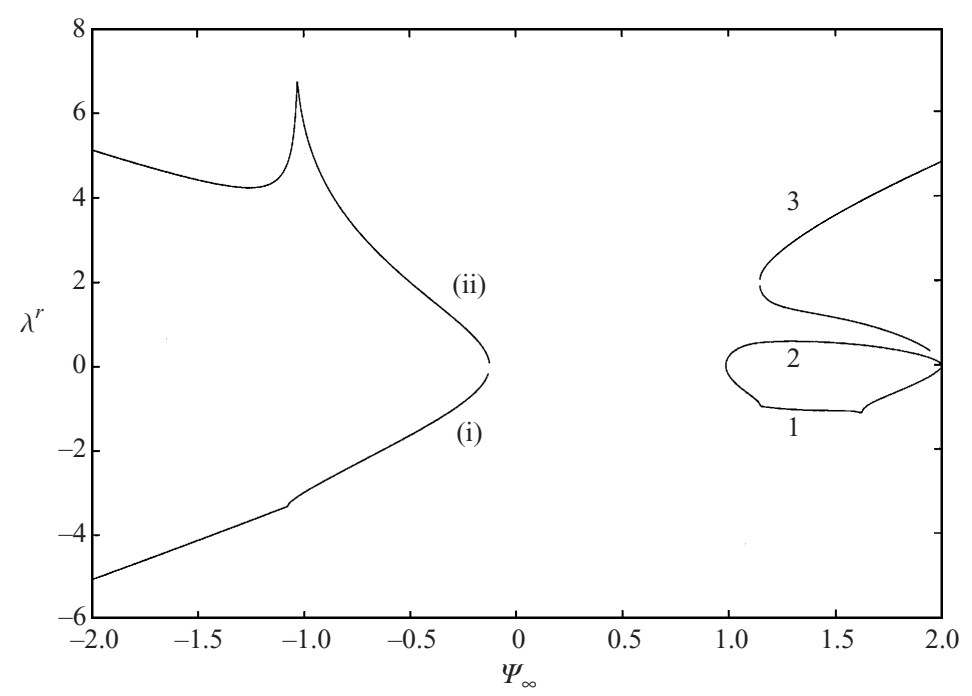

FIGURE 11. Variation of spatial eigenvalues $\left(\lambda^{r}\right)$ with $\Psi_{\infty}, n=0$. Here the labels indicate the corresponding base flow solutions, as denoted in figure 3 .

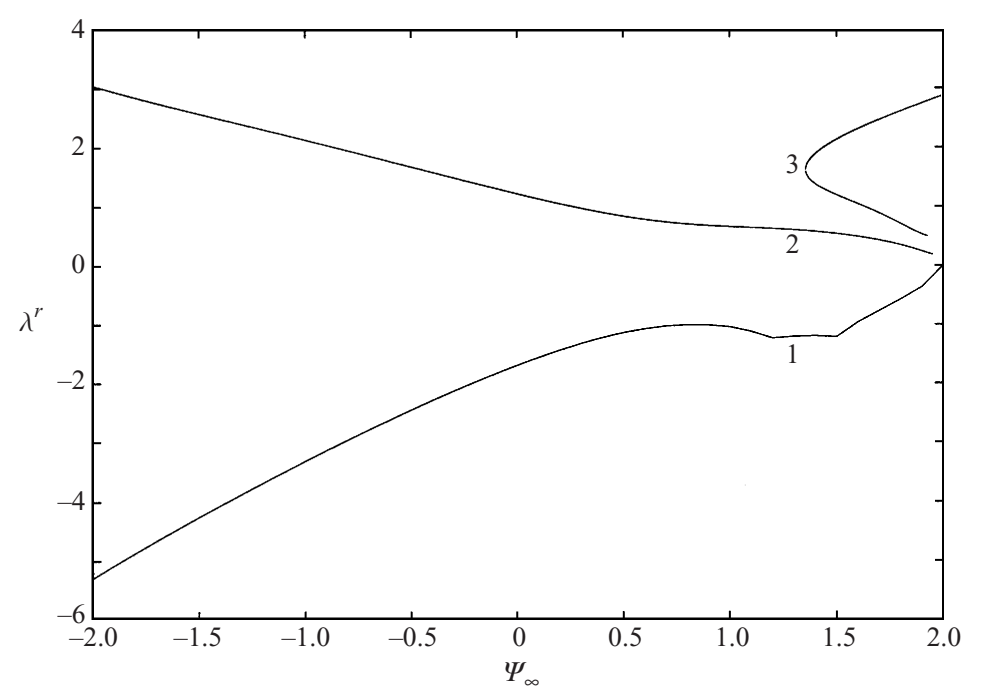

FIGURE 12. Variation of spatial eigenvalues $\left(\lambda^{r}\right)$ with $\Psi_{\infty}, n=1$. Here the labels indicate the corresponding base flow solutions, as denoted in figure 1.

difference methods) and standard shooting procedures (which involved an iterative procedure to determine each single value of $\lambda$ ). For consistency, we investigate the effect of these disturbances on just exponential base states. One may conjecture that an infinite number of discrete values of $\lambda$ exist and numerical evidence suggests that those modes of most importance are typically real. However in a few regimes, eigenvalues occurred as complex conjugate pairs, but in the following results we show just $\lambda^{r}=\operatorname{Re}\{\lambda\}$, which is responsible for controlling the spatial growth. A number of instances of complex conjugate eigenvalues coalescing to form two real eigenvalues were noted, and this process was responsible for the relatively rapid variation in $\lambda^{r}$ in some regions.

Results for the dependence of $\lambda^{r}$ on $\Psi_{\infty}$ for $n=0$ and $n=1$ are shown in figures 11 
and 12 respectively. In these figures we have presented just the most unstable/least stable eigenvalue (i.e. the largest value of $\lambda^{r}$ ), for each of the low-order exponential states outlined earlier in this paper, as labelled. One important observation is that for both values of $n$ considered, there exists at most one base state that possesses no positive $\lambda^{r}$ eigenvalues, and thus may be considered (in some sense) stable.

Calculations in the limit as $\Psi_{\infty} \rightarrow 2$ become increasingly difficult, but it is clear that eigenstates fall into two distinct categories, namely those such that $\lambda \rightarrow 0$ and others for which a finite limit is attained. As a consequence, some of the distributions in figures 11 and 12 (and in similar figures shown later) are not extended to $\Psi_{\infty}=2$ because the authors were unable to obtain reliable numerical results in this regime. These difficulties are caused by the development of disparate lengthscales, together with the increasing proximity of neighbouring eigenvalues, as revealed next.

Stability to exponential disturbances as $\Psi_{\infty} \rightarrow 2^{-}$

If we restrict attention to the stability of exponential base states to linearized perturbations that also approach free-stream conditions exponentially, then a discrete eigenvalue set is obtained. As we shall demonstrate, in the limit of $\Psi_{\infty} \rightarrow 2^{-}$, it is possible to make some analytical progress to show that an infinite sequence of near-neutral eigenvalues exist, although this may be in addition to further (unstable) eigenvalues.

Before introducing the appropriate description, we give a general overview of the behaviour. At the uppermost accumulation point shown in figure 1 (or indeed figure 3 ) for each solution an infinite discrete sequence of eigenvalues $\left\{\lambda_{k}\right\}$ exists, such that $\lambda_{k} \rightarrow 0(k=1,2,3, \ldots)$ as $\Psi_{\infty} \rightarrow 2^{-}$. However, at the lower accumulation point, although this same behaviour is found, in addition there are other eigenvalues that remain of order one and positive. Therefore, when $\Psi_{\infty}=2$ and $\delta^{*}=4$ (i.e. at the two accumulation points) the corresponding basic flow has an infinitely degenerate (neutral) eigenvalue (although other more 'dangerous' eigenvalues may also exist). An alternative view of the infinity of states in $\Psi_{\infty}<2$ (as shown in figures 1 and 3 ) is as a degenerate bifurcation process, where each of the infinite sequence of neutrally stable eigenmodes gives rise to a corresponding sequence of nonlinear states on perturbation away from $\Psi_{\infty}=2$.

The eigenvalue problem posed by the linearized stability equations, and our preceding analysis of the solution cascade are intimately connected. It is straightforward to show that when $\Psi_{\infty}=2-\varepsilon$ the far-field structure of the cross-flow perturbation is determined by

$$
\tilde{\psi}^{\prime \prime}+\left(\varepsilon \eta+\delta^{*}\right) \tilde{\psi}^{\prime}+(4-2 \varepsilon-2 \lambda) \tilde{\psi}=0,
$$

where $\delta^{*}=4-\varepsilon^{2 / 3} \delta_{j}$. This is valid for the $j$ th exponential base state, and we know from the description of $\S 4$ that $\delta_{j}=2^{-1 / 3}\left|\alpha_{j}\right|$, where $\alpha_{j}$ is the $j$ th zero of the Airy function.

A WKB analysis of the same form as given in $\S 4$ shows that, in this limit, an infinite sequence of near-neutral modes are present, such that $\lambda=\varepsilon^{2 / 3} \tilde{\lambda}_{k}$ with $k=1,2,3, \ldots$. Such an analysis proceeds as before, with a balance of the scaled eigenvalue $\tilde{\lambda}_{k}$ in the outer Airy region. Thus, for the $j$ th exponential solution branch, we find that

$$
\tilde{\lambda}_{k}=2^{-1 / 3}\left(\alpha_{k}-\alpha_{j}\right)
$$

for $k=1,2,3, \ldots \neq j$.

Thus, for the primary solution branch shown in figure 3 (denoted as branch 1) an infinite discrete sequence of eigenvalues can be found that all become neutral at 
$\Psi_{\infty}=2$, but are all stable when $\Psi_{\infty}=2-\varepsilon$. For branch 2 , a similar behaviour is found, but there is one eigenvalue $\left(\lambda_{1}\right)$ in the infinite set that approaches zero from above, leading to instability of this state in the region $\Psi_{\infty}<2$. Similarly, from (6.4) we see that the $j$ th branch will have $j-1$ unstable modes as $\Psi_{\infty}$ is decreased from $\Psi_{\infty}=2$, since there are obviously $j-1$ roots of the Airy equation larger than $\alpha_{j}$.

The near-neutral eigenvalues described above appear to be the entire set at the upper accumulation point shown in figure 1 (and 3); however this is not true at the lower accumulation point, near which finite unstable eigenvalues are also found as shown in figures 11 and 12 . These additional unstable eigenvalues cannot be captured by the expansion detailed above.

\section{Disturbance modes decaying algebraically into the free stream}

There is now an added twist to the overall picture, which has a connection with the preceding sections of the paper regarding the base flow, namely there seems no $a$ priori reason for necessarily enforcing exponential decay of the disturbance equations. Consequently, let us seek perturbation solutions which take the following form as $\eta \rightarrow \infty$ :

$$
(\tilde{u}, \tilde{\phi}, \tilde{\psi}) \rightarrow \eta^{-\alpha}(\hat{u}, \eta \hat{\phi}, \hat{\psi}),
$$

where we must of course demand that $\alpha>0$. Substitution of this form into (6.2) leads to the (general) conclusion that $\hat{u}=0$ (implying exponential decay of $\tilde{u}$ into the free stream) and

$$
\alpha=\frac{2\left(\Psi_{\infty}-\lambda\right)}{2-\Psi_{\infty}}
$$

(implying algebraic behaviour of $\tilde{\phi}$ and $\tilde{\psi}$ into the free stream). This result is applicable to both algebraic and exponential base states (the latter is not inconsistent, representing what may be regarded as free-stream vortical disturbances to boundarylayer flows; nevertheless, it would be difficult to justify the converse application of exponential modes to algebraic base states). Since we require $\Psi_{\infty} \leqslant 2$ for solutions to exist, the enforcement of decay of the eigensolutions as the free stream is approached (i.e. $\alpha<0$ ) leads to

$$
0<\lambda<\Psi_{\infty} \quad(\leqslant 2) ;
$$

the lower limit here arises from requiring disturbances that decay towards the leading edge, i.e. near $x=0$, the key point being that $\lambda$ can be arbitrarily specified, provided (6.7) is satisfied. The very important implication of this is that it is not necessary for $\lambda$ to actually be a (discrete) eigenvalue of the system (6.2), if the exponential decay restriction is removed. Consequently, this would suggest that eigensolutions which grow algebraically in the streamwise $(x)$ direction, but decay algebraically in the transverse $(\eta)$ direction are admissible (even for flows for which discrete, exponentially-decaying eigenvalues do not exist); this indicates a very broad range of applicability to this class of eigen-disturbance. Results for these 'eigenstates' to the Blasius-mode base flow, for three selected values of $\lambda$ are shown in figures $13(\tilde{u})$ and $14(\tilde{\psi})$, whilst the corresponding distributions for the other exponential $n=0$, $\Psi_{\infty}=1$ base state (termed the 'non-Blasius' state) are qualitatively similar and not shown here. The exponential behaviour of the $\tilde{u}$ distributions is readily apparent.

\subsection{Temporal disturbances}

In the case of $n=1$ it is completely justifiable within the exact nature of the similarity solution to investigate purely temporal disturbances to the base state. We 


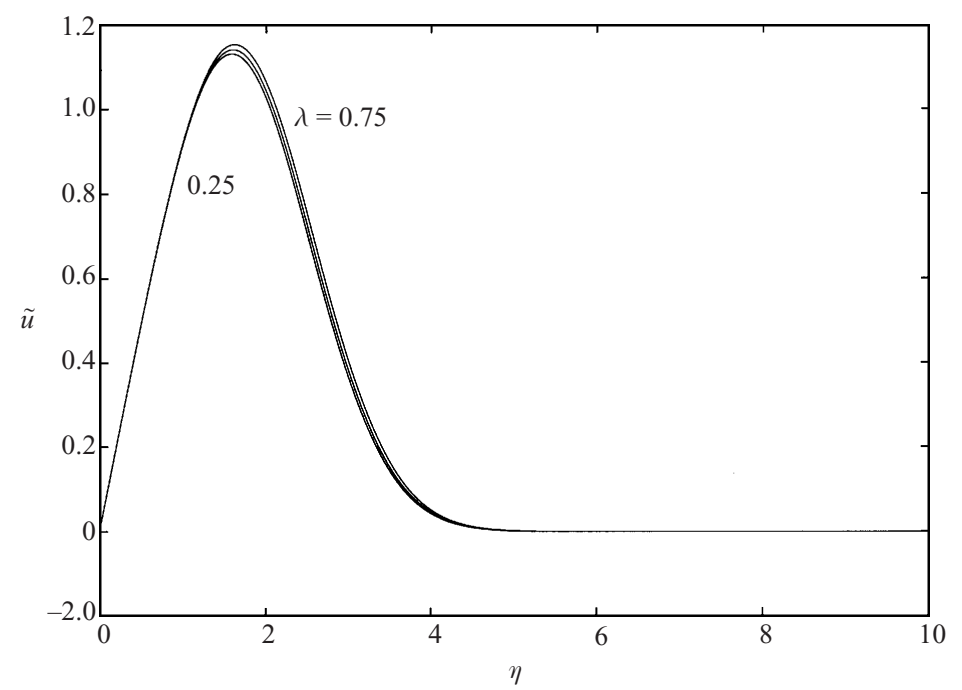

FIGURE 13. Variation of algebraically decaying $(\tilde{u})$ modes, Blasius base state.

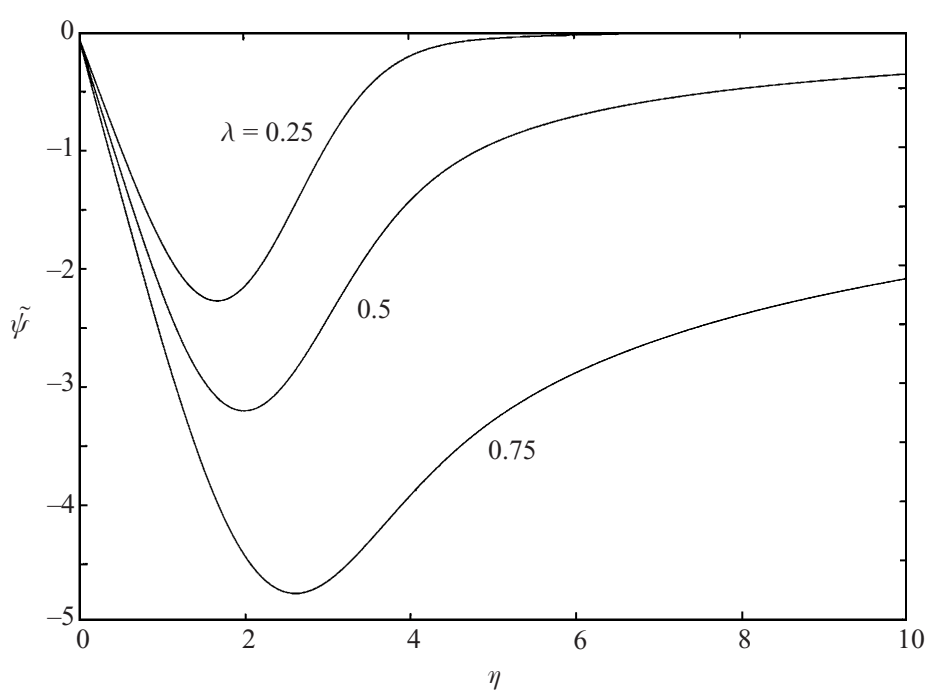

FIGURE 14. Variation of algebraically decaying $(\tilde{\psi})$ modes, Blasius base state.

may therefore seek solutions in the form

$$
U \rightarrow U+\hat{\varepsilon} \mathrm{e}^{\Omega t} \tilde{u}(\eta)+\cdots, \quad \Phi \rightarrow \Phi+\hat{\varepsilon} \mathrm{e}^{\Omega t} \tilde{\phi}(\eta)+\cdots, \quad \Psi \rightarrow \Psi+\hat{\varepsilon} \mathrm{e}^{\Omega t} \tilde{\psi}(\eta)+\cdots,
$$

assuming the amplitude parameter $|\hat{\varepsilon}| \ll 1$. To gain a simple heuristic estimate for the effect of temporal disturbances on the flow for other values of $n$ (in particular $n=0$ ) we also adopted this form for the general case; a similar procedure was used by Ridha (1992) (the proper procedure was described by Duck \& Dry 2001).

The characteristics described above for the spatial $(\lambda)$ eigenvalues are mimicked in their temporal counterparts (and therefore no numerical results are presented in the interests of brevity), with the majority of eigenvalues being real, just one base state being stable, corresponding to that with the largest streamwise wall shear stress (for example state 1 in figure 1 ). We note also the presence of coalescing eigenmodes in 
the limit $\Psi_{\infty} \rightarrow 2$. In fact, the near-neutral eigenmodes in this limit are also described by (6.4), but with $\tilde{\lambda}_{k}$ replaced by $\tilde{\Omega}_{k}$, where $\Omega=\varepsilon^{2 / 3} \tilde{\Omega}_{k}$. This previous description also applies to these temporal modes since for near-neutral eigenvalues, the analysis focuses on an outer scaled region of the boundary layer for which $U-1$ and $\tilde{u}$ are exponentially small, and in this region the two sets of (spatial/temporal) linearized disturbance equations are identical.

Just as with the spatial modes described in $\S 6.1$, it is again possible to relax the exponential decay into the free stream, and instead demand solutions of the form (6.5). This in turn leads us to similar results, but with $\lambda$ in (6.6) replaced by $\Omega$, where the same limits imposed on $\lambda$ in (6.7) are also applicable here for $\Omega(\tilde{u}$ again decays exponentially to the free-stream conditions). The consequence of this is that a continuum of temporally growing disturbance states can exist, even on base states for which discrete, temporally growing, exponential eigensolutions do not exist.

To conclude, therefore, it does appear that if the disturbance field is not subject to exponential decay, but merely algebraic decay, then unstable (temporal or spatial) modes are quite possible. In this respect there is an interesting analogy with the work of Timoshin (1995) who showed that all three-dimensional boundary layers are susceptible to short-wavelength, temporal, viscous instabilities (although in Timoshin's case the analysis was based on classical Orr-Sommerfeld theory, with imposed exponential decay).

\section{Discussion}

In this work we have re-investigated a generalization of a classical problem in fluid dynamics, in particular a class of self-similar boundary-layer flows applicable close to a line of symmetry. The solutions we present are parameterized by a 'cross-flow' parameter, $\Psi_{\infty}$, and the Falkner-Skan/pressure-gradient parameter $n$. Such flows have been discussed in some detail (in the case $n=1$ ), perhaps most notably by Howarth (1951), Davey (1961), Davey \& Schofield (1967) and Schofield \& Davey (1967).

From the past analyses of the $n=1$ case, it is known that the equations possess a continuum of states. Furthermore, under the requirement that the solutions also have a faster than algebraic approach to the free-stream conditions, it has been shown by Davey \& Schofield (1967) that the primary solution branch has an alternative, as denoted by branch 2 in figure 1 . For $\Psi_{\infty}>0$ these exponential modes are just two solutions in a continuum of states that, in general, will possess an algebraic component in the far field. Furthermore, the branch 2 solution of Davey \& Schofield (1967) is in fact just one of an infinity of such states. Furthermore a continuum of states that contain only exponentially decaying far-field behaviour exists for $\Psi_{\infty}=2$. How this continuum of exponentially decaying states could be perturbed to $\Psi_{\infty}<2$ and its relationship to the continuum of algebraic states was not addressed in previous work.

In this work we show that the solution structure is even more complex than previously assumed. Not only may several disconnected continua of states exist, but there remain embedded within the continua an infinity of states that do possess a faster than algebraic approach to the free-stream conditions; that is, they can be associated with an irrotational far-field flow. These embedded exponential modes appear as a cascade of states, analogous to the development of energy eigenstates in model problems in quantum mechanics, although more complex matching constraints are required owing to the semi-infinite nature of the problem under consideration here. It is through this cascade of exponential modes that the continua of exponential 
states located at $\Psi_{\infty}=2$ are connected (smoothly) to a discrete (but infinite) set of exponentially decaying solutions in $\Psi_{\infty}<2$, which are in turn interconnected by states that possess some quantity of algebraic decay in the far field. Furthermore, all these properties, although most easily placed in the context of past results using the particular case of $n=1$, are in fact seen to be generic features of the general boundary-layer equations.

Having shown a complex solution structure, with either continuous or discrete non-uniqueness (depending on ones willingness to discard the algebraically decaying states) a comment regarding the practical usefulness of such additional solutions must be made. As was noted with regard to the branch 2 solution presented by Davey \& Schofield (1967), many of the solutions are oscillatory and may be anticipated to be highly unstable and therefore of more mathematical than physical interest. This is especially true of the high-order branches or those states located in the limit of $\delta^{*} \rightarrow-\infty$ as described in $\S 5.1$. However, some of the solution branches are less easily discounted, in fact the analysis of $\S 6$ has noted that, in the case of the solutions shown in figure 3, the classical 'branch-1' solution can be viewed as stable whilst the alternative 'branch-2' state is an unstable solution with respect to a class of disturbances that develop in the streamwise direction (note that the branch2 solution includes the two-dimensional Blasius state). Nevertheless, it is far from clear, in general, what role the additional solutions play when, for example, $\Psi_{\infty}=2$ and the continuum consists of only exponentially decaying modes, or indeed what influence a small amount of free-stream vorticity could have on the boundary-layer structure. Some further work in this area is required to answer these questions and may be of particular interest near the accumulation point found at the saddle point of attachment (that is, $n=1$ and $\Psi_{\infty}=2$ ). Early analyses of the saddle point of attachment flow made an ad hoc assumption that the only relevant state in the continuum is that which approaches the appropriate edge conditions most rapidly (i.e. the fastest decaying exponential mode). The later work of Hall et al. (1992) highlighted that there is clearly no reason for making such an assumption, although it was shown that the fastest decaying state may have some implications for delineating stability boundaries of solutions within the continuum in their class of flow.

This study has been very much motivated by the effect of free-stream turbulence; what is most apparent is that the removal of the restriction of irrotationality in the external flow can have profound consequences, permitting a considerably broader class of boundary-layer solution. Over the years a number of studies have been made with respect to free-stream vorticity and its effects on boundary layers. This includes the work of Lighthill (1956), Toomre (1960), Crow (1966), Goldstein, Leib \& Cowley (1992), in addition to the previously mentioned work of Dhanak \& Stuart (1995). Obviously free-stream turbulence/vorticity can take many different forms, and we note that the form in this paper is somewhat different to that considered in the past, on account of the algebraic decay out of the boundary layers. We note that a number of the previously studies are characterized by having logarithmic behaviour, whilst Dhanak \& Stuart (1995) of course considered algebraically growing vorticity (but of small amplitude) towards the edge of the boundary layer.

The question of solution selection/stability has been raised in $\S 6$, where it is shown that, on assuming that the disturbance field possesses the same symmetries as the base flow, the primary base state is the most 'feasible' if one restricts disturbance modes to decay exponentially into the external flow. In some respects, since the primary stable state is that for which $U_{\eta}(0)$ is largest, one might view this as adding some quantitative weight to the assumption of Davey \& Schofield (1967), namely that the 
most significant state is that which approaches the free-stream conditions more rapidly than neighbouring states. A further caveat must also be noted, for if exponential decay of the disturbance field is relaxed (by the weaker requirement of algebraic decay), then all states may support a disturbance field that grows algebraically in the streamwise $(x)$ direction. Regardless of the cause of the growth, Duck (2002) has shown that nonlinear effects may then result in solution breakdown, with important physical implications, perhaps linked to transition. Broadening this discussion, it may initially seem surprising that so much non-uniqueness is present in our relatively simple system. However, we take the view that it is perhaps more surprising to have uniqueness. Other, well-known, relatively simple families of solution which exhibit gross non-uniqueness include those of Jeffery-Hamel and von Kármán rotating disk flows. The persistence of multiple (realistic) states in a truncated domain remains an open question.

As kindly pointed out by a referee, if the outer flow is inviscid, then the external vorticity will be conserved. A consequence of this is that if the boundary layer is of the exponential type, involving zero vorticity within the free stream, then it must remain so for all time. Equally, if vorticity is initially present, then it will remain so for all time. However (and this point may be invoked with respect to our arguments regarding algebraic disturbances imposed upon exponential states, for example), if the free stream is not inviscid (for example through the action of wakes or other viscous effects emanating from leading-edge devices), then clearly the Kelvin/Helmholtz inviscid results no longer apply, and the above restrictions/constraints are not applicable.

We have noted that the two-dimensional Falkner-Skan solutions are a subset of the three-dimensional flow considered here. In the Appendix we have drawn some parallels regarding the presence of a continuum of algebraic modes and cascade of exponential states in the Falkner-Skan equation, with those found in our more general three-dimensional problem. The general Falkner-Skan system has attracted a great deal of interest, most recently in terms of the manner in which cascades of periodic states are generated and destroyed as the Falkner-Skan parameter is increased, see for example, Hastings \& Troy (1988), Botta, Hut \& Veldman (1986) and Swinnerton-Dyer \& Sparrow (1995). One might anticipate that such solutions will also exist in the three-dimensional states studied here.

The authors would like to acknowledge the contribution of E. J. Watson for comments regarding the papers of A. Davey and D. Schofield. S. R. S. acknowledges the receipt of an EPSRC studentship.

\section{Appendix. Falkner-Skan solutions in an adverse pressure gradient}

We have already noted that in the case of $\Psi_{\infty}=(1-n)$ the well-known twodimensional Falkner-Skan solutions are a subset of our more general system (2.3). Substitution of $\Psi(\eta) \equiv(1-n) U(\eta)$ reduces $(2.3)$ to

$$
\begin{gathered}
U^{\prime \prime}+\Phi U^{\prime}+2 n\left(1-U^{2}\right)=0, \\
\Phi^{\prime}=(1+n) U .
\end{gathered}
$$

Eliminating $U$ in favour of $\Phi$, followed by the substitutions $f(z)=(1+n)^{1 / 2} \Phi(\eta)$ where $z=\eta(1+n)^{1 / 2}$, leads to

$$
f^{\prime \prime \prime}+f f^{\prime \prime}+\frac{2 n}{1+n}\left[1-\left(f^{\prime}\right)^{2}\right]=0,
$$



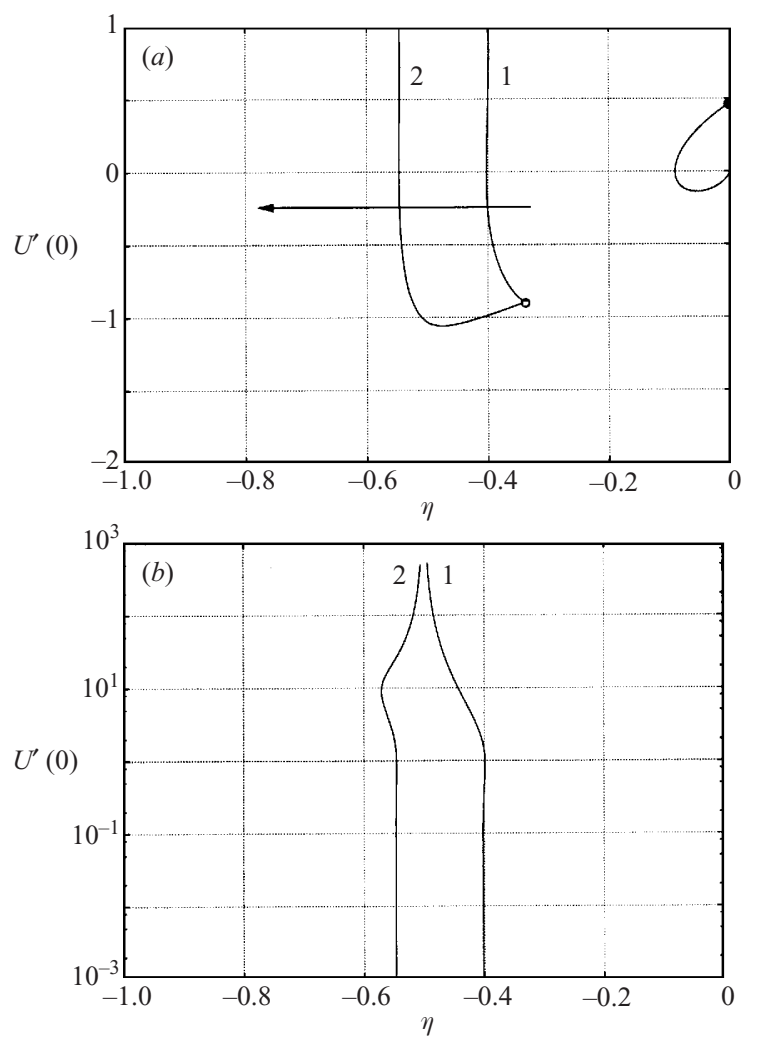

Figure 15. The Falkner-Skan solutions for $n<0$ that possess an exponential approach to the free-stream conditions. ( $a$ ) The Blasius solution is shown by the filled circle, and the open circle at $n=-1 / 3$ denotes one of a pair of terminal points for the infinity states that occur as $n \rightarrow-1^{+}$(as indicated by the arrow.) (b) The continuation of the first two branches to higher values of $U^{\prime}(0)$ (over a logarithmic scale). The other terminal point is found at $n=-1 / 2$.

which is the standard form of the Falkner-Skan equation following a further substitution for the Hartree parameter $\beta=2 n /(1+n)$.

The Blasius solution at $n=0$ can be continued to negative values of $n$ up to a fold at $n=n_{f} \approx-0.0904$ (see figure 15). No solutions have been located in the region $n \in\left[-1 / 3, n_{f}\right]$. In the far field $(\eta \gg 1)$ of $(\mathrm{A} 1)$, on writing $U=1+u(\eta)(|u| \ll 1)$ it is straightforward to show that

$$
u^{\prime \prime}+\left[(1+n) \eta+\delta^{*}\right] u^{\prime}-4 n u=0 .
$$

Clearly, (A 4) in the limit of $n \rightarrow-1^{+}$is analogous to $(2.8 b)$ as $\Psi_{e} \rightarrow 2^{-}$. The behaviour of (A 4) is essentially as given by (2.10) following a suitable substitution. Numerical results for the low-order exponential solution branches in $n \leqslant 0$ are shown in figure 15; results of this form were first presented by Libby \& Liu (1967), Oskam $\&$ Veldman (1982) and Brauner, Laine \& Nicolaenko (1982).

The limit of $n \rightarrow-1$ is equivalent to $\beta \rightarrow-\infty$ in terms of the Hartree parameter, and Troy (1979) has demonstrated that an infinite discrete sequence of solutions exist (with exponential decay) at $\beta=\left\{\beta_{j}\right\}(j=1,2, \ldots)$. In figure 15 only the first two solutions of this sequence are given. It becomes increasingly difficult to distinguish numerically between the exponential states and the algebraically decaying states as $n$ decreases towards $n=-1$. 
In general, there is an infinite sequence of solutions for $n \in(-1,-1 / 3)$. However, the terminal points of this sequence of solutions differ from that presented for the three-dimensional states described in $\S 4$. For $n=-1$, only non-decaying oscillatory profiles are possible and rather than a pair of terminal points existing at this limiting value (as was found to occur at $\Psi_{\infty}=2$ in the three-dimensional case) we find that the cascade of states terminates with two differing singular structures at $n=-1 / 2$ and $n=-1 / 3$.

The work of Oskam \& Veldman (1982) first proposed that the infinite, discrete, set of exponential modes originate from a single singular structure (as denoted by the open circle in figure 15). Furthermore, Brauner et al. (1982) have described the limit of $f^{\prime \prime}(0) \rightarrow \gamma$ with fixed $n=-1 / 3$, where $\gamma \approx-1.0863$ is a critical value determined in their analysis.

The limit $U^{\prime}(0) \rightarrow \infty$

We note that there appears to be a gap in the Falkner-Skan literature associated with the cascade of exponential states in the limit of $n \rightarrow-1^{+}$. There is an infinity of states to be found, which accumulate at one extreme with a singular structure as $n \rightarrow-1 / 3$ and, as we shall describe below, have a further accumulation point at $n=-1 / 2$. This infinity of solution branches stretches from $U^{\prime}(0)=+\infty$ at $n=-1 / 2$ to $n=-1 / 3$ at a critical value of $U^{\prime}(0)=(1+n)^{1 / 2} \gamma$ (where $\gamma \approx 1.0863$ ), and is illustrated for the first two exponential modes in figure 15 .

For large values of $U^{\prime}(0)$, prompted by numerical solutions, it is straightforward to formulate an appropriate asymptotic description of the form

$$
U=U_{0}(\hat{\eta}) \varepsilon^{-2 / 3}+\cdots, \quad \Phi=\Phi_{0}(\hat{\eta}) \varepsilon^{-1 / 3}+\cdots,
$$

in an inner region defined by $\hat{\eta}=\eta / \varepsilon^{1 / 3}=O(1)$, where $0<\varepsilon=1 / U^{\prime}(0)$. This description is to be valid near a critical value of $n=n_{0}+O\left(\varepsilon^{2 / 3}\right)$, where $n_{0}$ is to be determined.

The leading-order system in this inner region is simply the full Falkner-Skan system but with the absence of a pressure gradient term and boundary conditions of the form

$$
\Phi_{0}(0)=U_{0}(0)=0, \quad U_{0}^{\prime}(0)=1, \quad U_{0}(\infty)=0 .
$$

One can view this inner-layer system as providing a nonlinear eigenvalue problem for the unknown Falkner-Skan parameter $n_{0}$. However, it is simple to observe that the inner-layer equations, in the absence of any pressure gradient term (since it remains $O(1)$, and therefore does not appear at leading order) is simply the planar wall jet flow first discussed by Glauert (1956). Following Glauert's argument with a change in his notation of $\alpha=-2 n_{0} /\left(1+n_{0}\right)$, we find that such a solution can only exist for $n_{0}=-1 / 2$. Physically, we are stating that there is a near-wall region that is, in essence, a planar wall jet described by the solution of Glauert.

The discrete non-uniqueness of this limit occurs in an outer shear layer that is $O(1)$ in width but displaced $O\left(\delta^{-1 / 3}\right)$ from the boundary $\eta=0$. In the limit of $\hat{\eta} \rightarrow \infty$ (moving out from the thin inner region near the boundary) we find that $U \rightarrow-1$ for all solution branches apart from the first (for which $U \rightarrow 1$, and no outer shear-layer region exists). For branches $2,3, \ldots$ an outer region exists in which there is a transition from $U=-1$ to $U=1$. The equations in this outer layer are the full Falkner-Skan equations, as given by (A 1 ), but with $n=n_{0}=1 / 2$ and with boundary conditions of $U \rightarrow \pm 1$ as $\tilde{\eta} \rightarrow \pm \infty$, where $\eta=\eta_{0} / \varepsilon^{1 / 3}+\tilde{\eta}$. Numerical evidence points to the existence of a discrete infinity of solutions to the outer shear layer that possess an 
exponential approach to $U=1$ as $\tilde{\eta} \rightarrow \infty$, although we do not attempt to prove this rigorously.

\section{REFERENCES}

Ackerberg, R. C. 1965 The viscous incompressible flow inside a cone. J. Fluid Mech. 21, 47.

Botta, E. F. F., Hut, F. J. \& Veldman, A. E. P. 1986 The role of periodic solutions in the Falkner-Skan problem for $\lambda>0$. J. Engng Maths 20, 81 .

Brauner, C. M., Laine, Cl. \& Nicolaenko, B. 1982 Further solutions of the Falkner-Skan equation for $\beta=-1$ and $\gamma=0$. Mathematika 29, 231.

Brown, S. N. \& StewARTSON, K. 1965 On similarity solutions of the boundary-layer equations with algebraic decay. J. Fluid Mech. 23, 673.

Craven, A. H. \& Peletier, L. A. 1972 On the uniqueness of solutions of the Falkner-Skan equation. Mathematika 19, 135.

Crow, S. C. 1966 The spanwise perturbation of two-dimensional boundary layers. J. Fluid Mech. 24, 153.

DaveY, A. 1961 Boundary-layer flow at a saddle point of attachment. J. Fluid Mech. 10, 593.

DaveY, A. \& Schofield, D. 1967 Three-dimensional flow near a two-dimensional stagnation point. J. Fluid Mech. 28, 149 .

DhanaK, M. R. \& DuCK, P. W. 1997 The effects of freestream pressure gradient on a corner boundary layer. Proc. Roy. Soc. Lond. A 453, 1793.

DhanaK, M. R. \& StUART, J. T. 1995 Distortion of the stagnation-point flow due to cross-stream vorticity in the external flow. Phil. Trans. R. Soc. Lond. A 352, 443.

Drazin, P. G. \& Reid, W. H. 1981 Hydrodynamic Stability. Cambridge University Press.

Duck, P. W. 2002 Streamwise algebraic growth (and breakdown) in three-dimensional boundary layers. Theor. Comp. Fluid Dyn. (submitted).

DuCK, P. W. \& DrY, S. L. 2001 On a class of unsteady, non-parallel, three-dimensional disturbances. J. Fluid Mech. 441, 31.

Duck, P. W., Stow, S. R. \& Dhanak, M. R. 1999 Non-similarity solutions to the corner boundarylayer equations (and the effects of wall transpiration). J. Fluid Mech. 400, 125.

Duck, P. W., Stow, S. R. \& Dhanak, M. R. 2000 Boundary-layer flow along a ridge: alternatives to the Falkner-Skan solutions. Phil. Trans. R. Soc. Lond. 358, 3075.

Glauert, M. B. 1956 The wall jet. J. Fluid Mech. 1, 625.

Goldstein, M. E., Leib, S. J. \& Cowley, S. J. 1992 Distortion of a flat-plate boundary layer by free-stream vorticity normal to the plate. J. Fluid Mech. 237, 231.

GoldsTEIN, S. 1965 On backward boundary layers and flow in converging passages. J. Fluid Mech. 21, 33.

Hall, P., Balakumar, P. \& Papageorgiou, D. 1992 On a class of unsteady three-dimensional Navier-Stokes solutions relevant to rotating disc flows: threshold amplitudes and finite-time singularities. J. Fluid Mech. 238, 297.

Hastings, S. P. \& Troy, W. 1988 Oscillating solutions of the Falkner-Skan equation for positive beta. J. Diffl Equat. 71, 123.

HewitT, R. E. \& DUCK, P. W. 2000 Non-axisymmetric rotating-disk flows: nonlinear travelling-wave states. J. Fluid Mech. 413, 287.

HewitT, R. E., DuCK, P. W. \& Foster M. R. 1999 Steady boundary-layer solutions for a swirling stratified fluid in a rotating cone. J. Fluid Mech. 384, 339.

Howarth, L. 1951 The boundary layer in three-dimensional flow. Part II. The stagnation point. Phil. Mag. 42(7), 1433.

KaChanov, Y. S. 1994 Physical mechanisms of laminar-boundary-layer transition. Annu. Rev. Fluid Mech. 26, 411.

LibBy, P. A. \& LiU, T. M. 1967 Further solutions of the Falkner-Skan equation. AIAA J. 5, 1040.

Lighthill, M. J. 1956 Drift. J. Fluid Mech. 1, 31.

LUCHINI, P. 1996 Reynolds-number-independent instability of the boundary layer over a flat surface. J. Fluid Mech. 404, 289.

MaLIK, M. 1986 The neutral curve for stationary disturbances in rotating disk flow J. Fluid Mech. 164,275 . 
Oskam, B. \& Veldman, A. E. P. 1982 Branching of the Falkner-Skan solutions for $\lambda<0$. J. Engng Maths 16, 295.

RidHA, A. 1992 On the dual solutions associated with boundary-layer equations in a corner. $J$. Engng Maths 26, 525.

Schofield, D. \& DAVEY, A. 1967 Dual solutions of the boundary-layer equations at a point of attachment. J. Fluid Mech. 30, 809.

Stow, S. R. 1999 On a class of three-dimensional boundary-layer flows. PhD Thesis, University of Manchester.

Swinnerton-Dyer, H. P. F. \& Sparrow, C. 1995 The Falkner-Skan equation I: The creation of strange invaariant sets. J. Diff Equat. 119, 336.

Timoshin, S. N. 1995 On short-wavelength instabilities in three-dimensional classical boundary layers. Eur. J. Mech. B/Fluids 14, 409.

Toomre, A. 1960 The viscous secondary flow ahead of an infinite cylinder in a uniform parallel shear flow. J. Fluid Mech. 7, 145.

Troy, W. C. 1979 Non-monotonic solutions to the Falkner-Skan boundary layer equation. Q. Appl. Maths 37, 157. 